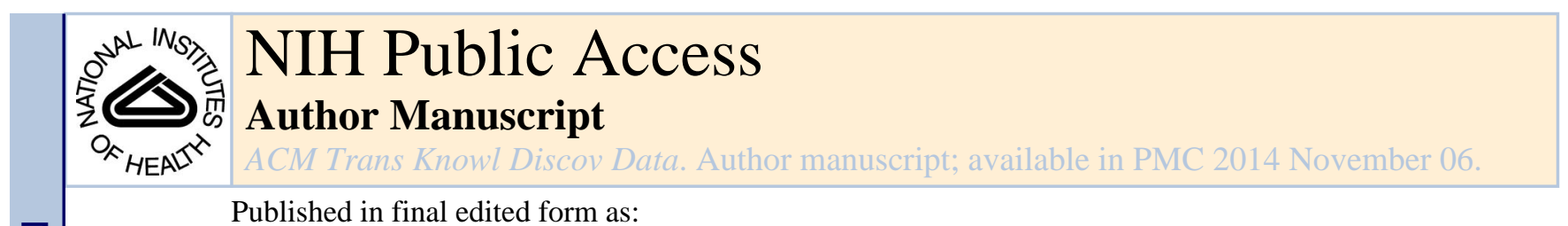

Published in final edited form as:

ACM Trans Knowl Discov Data. 2014 February ; 8(1): . doi:10.1145/2518176.

\title{
Scalable and Axiomatic Ranking of Network Role Similarity
}

\author{
Ruoming Jin, \\ Kent State University \\ Victor E. Lee ${ }^{1}$, and \\ John Carroll University \\ Longjie $\mathrm{Li}^{2}$ \\ Lanzhou University
}

\begin{abstract}
A key task in analyzing social networks and other complex networks is role analysis: describing and categorizing nodes according to how they interact with other nodes. Two nodes have the same role if they interact with equivalent sets of neighbors. The most fundamental role equivalence is automorphic equivalence. Unfortunately, the fastest algorithms known for graph automorphism are nonpolynomial. Moreover, since exact equivalence is rare, a more meaningful task is measuring the role similarity between any two nodes. This task is closely related to the structural or link-based similarity problem that SimRank addresses. However, SimRank and other existing similarity measures are not sufficient because they do not guarantee to recognize automorphically or structurally equivalent nodes. This paper makes two contributions. First, we present and justify several axiomatic properties necessary for a role similarity measure or metric. Second, we present RoleSim, a new similarity metric which satisfies these axioms and which can be computed with a simple iterative algorithm. We rigorously prove that RoleSim satisfies all these axiomatic properties. We also introduce Iceberg RoleSim, a scalable algorithm which discovers all pairs with RoleSim scores above a user-defined threshold $\theta$. We demonstrate the interpretative power of RoleSim on both both synthetic and real datasets.
\end{abstract}

\section{General Terms}

Theory; Algorithms; Measurement; Experimentation

\footnotetext{
${ }_{1}^{1}$ Research initiated while affiliated with Kent State University

${ }^{2}$ Visiting Scholar at Kent State University

Permission to make digital or hard copies of part or all of this work for personal or classroom use is granted without fee provided that copies are not made or distributed for profit or commercial advantage and that copies show this notice on the first page or initial screen of a display along with the full citation. Copyrights for components of this work owned by others than ACM must be honored. Abstracting with credit is permitted. To copy otherwise, to republish, to post on servers, to redistribute to lists, or to use any component of this work in other works requires prior specific permission and/or a fee. Permissions may be requested from Publications Dept., ACM, Inc., 2 Penn Plaza, Suite 701, New York, NY 10121-0701 USA, fax +1 (212) 869-0481, or permissions@acm.org.
}

ELECTRONIC APPENDIX

The electronic appendix for this article can be accessed in the ACM Digital Library. 


\section{Additional Key Words and Phrases}

Complex network; Social network; Vertex similarity; Role similarity; Ranking; Automorphic equivalence

\section{INTRODUCTION}

In social science, it is well-established that individual agents tend to play roles or assume positions within their interaction network. For instance, in a university, each individual can be classified into the position of faculty member, administration, staff, or student. Indeed, role discovery is a major research subject in classical social science [Wasserman and Faust 1994]. Interestingly, recent studies have found that roles not only appear in other types of networks, including food webs [Luczkovich et al. 2003], world trade [Hafner-Burton et al. 2009], and even software systems [Dragan et al. 2009], but also can help to predict node functionality within their domains. For example, in a protein interaction network, proteins with similar roles tend to serve similar metabolic functions. Thus, if we know the function of one protein, we can predict that all other proteins having a similar role would also have similar function [Holme and Huss 2005]. In other cases, such as online social networks, there are no a priori role categories. The classification must be learned based on the interaction patterns.

Role discovery is complementary to graph clustering, an important tool for analyzing network structures. Graph clustering attempts to decompose a graph into densely connected components. It produces a high level structural model consisting of a small number of "cluster-nodes" and the "super-edges" between these cluster-nodes. The clustering scheme inevitably overlooks and oversimplifies the interaction patterns of the individual nodes. In reality, the nodes within a cluster may take very different roles: some of them may serve as the core of the clusters, some may be peripheral nodes, and some serve as the connectors to link between clusters. At the same time, nodes located in different clusters might play similar roles. Furthermore, even when a network lacks modularity structure, such as in a simple hierarchical structure, roles can still characterize the interaction patterns of each node. Hence, roles provide an orthogonal abstraction for simplifying and highlighting the complex interactions among nodes.

A central question in studying the roles in a network system is how to define role similarity. In particular, how can we rank two nodes' role similarity in terms of their interaction patterns? Despite its vital importance for network analysis and decades of work by social scientists, joined recently by computer scientists, no satisfactory metric for role similarity has yet emerged. A key issue is the encapsulation of graph automorphism into a role similarity metric: if two nodes are automorphically equivalent, then they should share the same role and their role similarity should be maximal. From a network topology viewpoint, automorphic nodes have equivalent surroundings, so one can replace the other. Figure 1 illustrates a graph with nodes $S 1$ and $J 1$ being automorphically equivalent.

The traditional social science approach for role analysis has been to define suitable mathematical equivalence relations for nodes so they can be partitioned into equivalence 
classes (roles). An essential property of these equivalences is that they should positively confirm automorphic equivalence, i.e., if any two nodes are automorphic, then they are roleequivalent. (The converse is not necessarily true.) Confirming automorphism is an instance of verifying a solution, which is often algorithmically less complex than discovering a solution. Thus though there is no known polynomial-time algorithm for discovering graph automorphism, ${ }^{3}$ role equivalence algorithms [Batagelj et al. 1992; Borgatti and Everett 1993; Sparrow 1993] can still guarantee to satisfy the aforementioned automorphism confirmation property. These equivalence rules also directly correspond to the aforementioned coloration.

However, by relying on strict equivalence rules, these role modeling schemes can produce only binary similarity metrics: two nodes are either equivalent (similarity $=1$ ) or not (similarity $=0$ ). In real-world networks, usually only a very small portion of the node-pairs would satisfy an equivalence criteria [MacArthur et al. 2008] and among those, many are simply trivially equivalent (such as singletons or children of the same parent). In addition, strict rule-based equivalence is not robust with respect to network noise, such as falsepositive or false-negative interactions. Thus, it is desirable in many real world applications to rank node-pairs by their degree of similarity or provide a real-valued node similarity metric.

Recent research works have proposed various measures of node similarity based on similarity of interactions. In [Leicht et al. 2005], $a$ accrues similarity to $b$ if $a$ has a neighbor which is similar to $b$. This assumes that neighbors ( $a$ and its neighhors) should be somewhat similar; however, roles should be class-based, not proximity-based. Jeh's SimRank [Jeh and Widom 2002] is based on the following principle: "two nodes are similar if they link to similar nodes". Mathematically, for any two different nodes $x$ and $y$, SimRank computes their similarity recursively according to the average similarity of all the neighbor pairs (a neighbor of $x$ paired with a neighbor of $y$ ). A single node has self-similarity value 1 . This is equivalent to the probability that two simultanous random walkers, starting at $x$ and $y$, will eventually meet. Most other node structural similarity measures [Antonellis et al. 2008; Fogaras and Rácz 2005; Li et al. 2009; Xi et al. 2005; Yin et al. 2006; Zhao et al. 2009] are variants of SimRank. Though SimRank seems to capture the intuition of the above recursive structural similarity, its random walk matching does not satisfy the basic graph automorphism condition. For example, in Figure 1, though $S 1$ and $J 1$ are automorphically equivalent, SimRank assigns them a value of 0.226. We discuss this further in Section 3.2. To our best knowledge, there is no available real-valued structural similarity measure satisfying the automorphic equivalence requirement. Since automorphic equivalence is a pivotal characteristic of the notion of role, its lack disqualifies these existing measures from serving as authentic role similarity measures.

Thus we have an open problem: Can we derive a real-valued role similarity measure or ranking which complies with the automorphic equivalence requirement? In this paper, we develop the first real-valued similarity measure to solve this problem. In addition, our measure is also a metric, i.e., it satisfies the triangle inequality. The key feature of our role

\footnotetext{
${ }^{3}$ The computational complexity of graph isomorphism and automorphism are still unproven to be either $P$ or $N P-$ Complete.
} 
similarity measure is a weighted generalization of the Jaccard coefficient to measure the neighborhood similarity between two nodes. Unlike SimRank, which considers the average similarity among all possible pairings of neighbors, our measure considers only those pairs in the optimal matching of their two neighbor sets which maximizes the targeted similarity function.

The paper is organized as follows: Section 2 provides a detailed review of the existing works on node similarity. Section 3 presents axiomatic properties of any real-valued similarity measure, including a requirement for automorphic equivalence. We also show that SimRank does not satisfy the automorphic equivalence requirement. Section 4 describes our RoleSim measure, its computation, and its correctness with respect to the axiomatic properties. Section 5 presents Iceberg RoleSim, a scalable algorithm which discovers all pairs with RoleSim scores above a user-defined threshold. Section 6 provides experimental validation and evaluation of our proposed approach for ranking the the role similarity between vertex pairs.

An earlier version of the paper [Jin et al. 2011] is published in KDD'11. This paper significantly extends that work and makes the following additional contributions: 1) we provide a detailed and extensive survey of the existing node similarity work (Section 2);2) we introduce a new Iceberg RoleSim to scale the existing RoleSim computation (Section 5); 3) we perform a detailed evaluation of Iceberg RoleSim to demonstrate its performance and scalability (Subsection 6.5); 4) we present a detailed case study comparing RoleSim with the state-of-the-art approaches using a co-author network (Subsection 6.6).

\section{RELATED WORK}

This section provides a survey of prior work relevant to our core problem of role similarity. The first section describes several formal definitions of role and role equivalence. The next section reviews existing work on role similarity. The remaining sections review numerous measures for the general problem of local structural similarity, considering first centralitybased measures and then link-based measures.

We take this opportunity to establish some symbolic notation to used here and in the remainder of this work. We use the terms graph and network interchangeably; the same is true for vertex and node. In most instances, we speak of networks and nodes, as this is the more common usage in the principal application domains of interest, but we revert to graph and vertex at times when speaking in a graph-theoretical sense.

We define a graph or network $G=(V, E)$ as a set of nodes $V$ and a set of connecting edges $E$ $\subseteq V \times V$. The neighbors of a node $v$ are those nodes which are joined directly to $v$ with an edge. The set of neighbors $N(v)=\{u \mid(u, v) \in E\}$. The degree of $v$ is $d_{v}=|N(v)|$. When discussing computational complexity, the number of vertices in a graph is $n=|V|$, and the number of edges is $m=|E|$. By default, we assume that edges are undirected, but all of the concepts and formulas in this work can be extended to directed graphs by computing scores using in-neighbors and out-neighbors separately and then combining the scores. 


\subsection{Role Equivalence}

Computing role similarity encompasses two more fundamental problems: what is a role, and how should we measure closeness to role? We use the following definition of role:

Definition 1. Role and Role Equivalence. A role is the set of relationships between an individual and others. In graph theory terms, the role of $v$ is the set of all edges incident to v. For an undirected graph: role $(v)=\{(u, v) \in E\}$. Two individuals fulfill equivalent roles if they have equivalent relationships.

For example, consider Figure 1, which depicts three siblings $\{S 1, J 1, L 1\}$, who are each a parent in a family. Each family has two parents and either two or three children. There are three types of relationships shown:

1. Spouse $\{\mathrm{S} 1-\mathrm{S} 2, \mathrm{~J} 1-\mathrm{J} 2, \mathrm{~L} 1-\mathrm{L} 2\}$

2. Parent-Child \{S1-S3, S1-S4, S2-S3, S2-S4, J1-J3, etc. $\}$

3. Sibling $\{$ S1-J1, S1-L1, J1-L1 $\}$

For simplicity, we do not show the sibling relationships in the younger generation.

Intuitively, S1 and J1 appear to be role-equivalent: each is a spouse, a parent of two children, and the sibling of two others. Note we have not labeled or colored the edges, only the nodes. For example, a parent-child relationship is defined by the two participating nodes, not by a pre-labeling of the edge. However, we do not know that the two ends represent a parent and a child, until we identify the roles. In general, even the nodes will not be labeled or colored in advance. We will begin only with a graph topology; the role equivalence discovery problem is to identify the colorings.

In social network analysis, the traditional approach for discovering role groups is to define a equivalence relation and to partition the actors into equivalence classes. Actors who fulfill the same role are equivalent. Over the years, four definitions have stood out. These four, in decreasing order of strictness, are structural equivalence, automorphic equivalence, equitable partition, and regular equivalence. Table 1 shows how these different definitions generate different roles from the same network.

- Structural Equivalence: Two actors are structurally equivalent if they interact with the same set of others [Lorrain and White 1971]. Mathematically, $u$ and $v$ are structurally equivalent if and only if $N(u)=N(v)$. For example, consider the extended family shown in Figure 1. $S 1, J 1$, and $L 1$ are siblings, $S 2, J 2$, and $L 2$ are spouses, and the remaining nodes are their children. Each family's children, $\{S 3$, $S 4\},\{J 3, J 4\}$, and $\{L 3, L 4, L 5\}$, form a nontrivial equivalence class. However, none of the parents can be grouped together via structural equivalence. Figure 2(a) illustrates this partitioning. Nodes with the same color are in the same class, except gray nodes represent singleton classes. Each gray node is its own class. This model is too strict to be useful for simplifying a large network and to discover meaningful roles. 
Automorphic Equivalence: Two actors (nodes) $u$ and $v$ are automorphically equivalent if there is an automorphism $\sigma$ of $G$ where $v=\sigma(u)$ [Borgatti and Everett 1992]. An automorphism $\sigma$ of a graph $G$ is a permutation of vertex set $V$ such that for any two nodes $u$ and $v,(u, v) \in E$ iff $(\sigma(u), \sigma(v)) \in E$. In social terms, $u$ and $v$ can swap names, along with possibly some other name swaps, while preserving all the actor-actor relationships. Let $\Gamma(G)$ be the group of all automorphisms of graph $G$. For any two nodes $u$ and $v$ in $G, u \equiv v$ if $u=\sigma(v)$ for some $\sigma \in \Gamma(G)$. Note that $\equiv$ is an equivalence relation on $V$; if $u \equiv v$ we say that $u$ is automorphically equivalent to $v$. The equivalence classes generated under $\Gamma(G)$ (or $\equiv$ ) are called orbits. The equivalence class for vertex $v \in V$ is called the orbit of $v$, and denoted as $\Delta(v)=\{\sigma(v) \in V, \sigma \in \Gamma(G)\}=\{u \mid u \equiv v\}$. Each orbit corresponds to a role in the automorphic equivalence. Understanding the importance of automorphic equivalence and applying it to role modeling was a major breakthrough in classical social network research. In our example Figure 1, from the topology alone, we cannot distinguish between the Smith family and the Jones family. The Lee family is distinct, because it has three children instead of two. Therefore, the equivalence classes are $\{S 1, J 1\},\{S 2, J 2\},\{S 3, S 4, J 3, J 4\},\{L 1\},\{L 2\}$, and $\{L 3, L 4, L 5\}$

(Figure 2(b)). Interestingly, automorphically equivalent classes must have equivalent indirect relations as well, such as equivalent in-laws and cousins. However, automorphic equivalence is hard to compute and still very strict.

- Exact Coloration (Equitable Partition): An exact coloration of graph $G$ assigns a color to each node, such that any two nodes share the same color iff they have the same number of neighbors of each color [Everett and Borgatti 1996]. Nodes of the same color form an equivalence class. An exact coloration is also referred to as equitable partition [Godsil and Royle 2001] and graph divisor [Cvetkovíc et al. 1998] and is often applied in the vertex classification/refinement for canonical labeling in a graph isomorphism test [Read and Corneil 1977; McKay 1981]. A graph may have several exact colorations; in general we seek the fewest colors. In our example, structural equivalence and automorphic equivalence offer two different exact colorations. Exact coloration relaxes automorphism by considering only immediate neighborhood equivalence, yet it still embodies a recursive aspect to role modeling.

- Regular Equivalence (Bisimulation): Two actors are regularly equivalent if they interact with the same variety of role classes, where class is recursively defined by regular equivalence [White and Reitz 1983]. Unlike automorphic equivalence and exact coloration, regular equivalence does not care about the cardinality of neighbor relationships, only whether they are nonzero. For example, using regular equivalence, all three families could be equivalent, with only three equivalence classes: sibling - parent $\{S 1, J 1, L 1\}$, spouse - parent $\{S 2, J 2, L 2\}$, and child (Figure 2(c)). Note that under regular equivalence, any two automorphically equivalent nodes may be merged into the same regular equivalence class. In computer science, the regular equivalence is often referred to as the bisimulation, which is widely used in automata and modal logic [Marx and Masuch 2003]. 


\subsection{Existing Role Similarity Measures}

We now move from strict equivalence to measuring similarity. There has been limited work on measuring role similarity. For structural equivalence, one can count how many neighbors they share, normalized by some factor. However, we noted in the previous section that structural similarity is too limiting for our interest.

Two algorithms for measuring the extent of regular equivalence are described in [Borgatti and Everett 1993]. However, the authors acknowledge "the lack of a theoretical rationale for the measure of similarity produced." The core of the problem lies not in their algorithms but in regular equivalence itself. Both regular equivalence and exact coloration are problematic because there may be more than one equivalence partitioning for a given graph. Indeed, for regular equivalence, every graph has two degenerate partitionings: (1) place all nodes in one class and (2) place each node in its own class (except structurally equivalence nodes may be in the same class). If one is measuring similarity, from which partition are you measuring the similarity?

To find the "best" regular partitioning, one can consider an information-theoretic or minimum description length (MDL) approach: group nodes into classes or blocks that approximately describe a true regular equivalence class membership. This is the blockmodeling approach [Batagelj et al. 1992; Doreian et al. 2005]. MDL blockmodeling tries to solve the following optimization problem: Assign $n$ nodes to $b$ blocks such that the aggregate cost of describing the block structure $O(b \log b)$ plus the cost of describing the difference between the appropriate block structure and the true structure is minimized. Heuristically, the problem is easier if the number of blocks $b$ is preset, but whether it is or not, the exact optimization problem is NP-hard. Furthermore, it does not truly address our problem: we want to know how similar are nodes at the individual level. Blockmodeling jumps ahead to a global partitioning problem and only provides a rough measure of distance.

\subsection{Structural Similarity Measures}

Due to the limited work in role similarity, we look at prior work for other types of structural similarity, namely, (1) centrality of a node with respect to the full graph and (2) link-based similarity. We will not consider density measures. Density has been well-studied in other works [Lee et al. 2010] and is not relevant to our definition of role similarity.

2.3.1. Similarity of Node Centrality-This section discusses properties of individual nodes, in the context of a network. By themselves, these properties are not similarity measures. However, the property values of two different nodes can be compared to produce a similarity measure. Node degree, closeness centrality, and betweenness centrality are three such measures. Degree counts the number of incident edges. In directed graphs, it can be divided into in-degree and out-degree. Closeness centrality is the average distance between a node $v$ and every other node in the graph. Betweenness centrality measures how frequently a node lies on the path between two other nodes [Freeman 1977]. These definitions, however, are too limited to encompass the concept of role. Role is not merely centrality of degree, closeness, or betweenness. It is any or all of these and possibly more; it is whatever makes the structural position of a node unique. 
2.3.2. Link Similarity-Another way that node structural similarity has been defined is in terms of link similarity. That is, how are two nodes connected to one another? One of the earliest measures of link similarity is bibliographical coupling [Kessler 1963]. This measures the similarity between two research publications by counting the number of works that are listed in both of their bibliographies. Co-citation [Small 1973] turns this around by counting the number of later works that cite both of the two original documents. As the size of a work's bibliography increases, the likelihood that it will contain a particular work increases. Therefore, a common normalization of these two measures is to divide the count by the number of distinct works cited.

We can form a citation graph, where each node is a document and a directed edge $(a, b)$ means that document $a$ cites document $b$. Let $I(a)$ and $O(a)$ be the in-neighbor set and outneighbor set of $a$, respectively. Let $I_{a}$ and $O_{b}$ be the in-degree and out-degree of $a$. Then, the normalized bibliographic coupling index is

$$
S_{b c}(a, b)=\frac{|O(a) \cap O(b)|}{|O(a) \cup O(b)|},
$$

and the normalized co-citation index is

$$
S_{c c}(a, b)=\frac{|I(a) \cap I(b)|}{|I(a) \cup I(b)|} .
$$

These are simply the Jaccard coefficients of the out-neighbor sets and in-neighbors sets, respectively.

These two are suitable for unweighted and directed graphs. If a graph is undirected, then the two measures are the same. Suppose we have a weighted graph, though. This could be an author-collaboration graph, where edge $(a, b)$ counts how many times author $a$ has worked with author $b$. Or, it could be a bipartite document-term graph, where edge $\left(d_{a}, t_{b}\right)$ counts the number of times that document $a$ uses term $b$. Assign to each node a feature vector. For a co-authorship graph, each author is a feature dimension; its feature vector is the set of edge weights to every other author. For a document-term bipartite graph, a document has a term vector, weighted according to term frequencies of the document. If we represent the graph as an adjacency matrix, then the feature vector of node $i$ is the $i_{\text {th }}$ row of the matrix.

Given this representation, the cosine between two objects is a convenient and meaningful measure. Identical documents have cosine of 1 , and documents with no features in common are orthogonal with cosine of 0 .

$$
S_{c i t}(a, b)=\frac{A \cdot B}{\|A\|\|B\|},
$$

where $A$ is the feature vector of node $a$.

A small modification to the denominator of Eq. 3, attributed to Tanimoto [Tanimoto 1958], maintains the overall behavior of the similarity function while aligning it with the Jaccard coefficient when the feature vectors are binary-valued: 


$$
S_{\text {tani }}(a, b)=\frac{A \cdot B}{\|A\|^{2}+\|B\|^{2}-A \cdot B}
$$

Schultz [Schultz and Liberman 1999] adapted the well-known TF-IDF query-document similarity measure to produce a term-weighted document-document similarity measure. Here, $A(t)$ is the frequency of term $t$ for object $a$, and $i d f(t)$ is the inverse document frequency for term $t$. More generally, it is the significance or importance of term $t$ appearing in a document.

$$
S_{w \cos }(a, b)=\frac{\sum_{t \in T} A(t) B(t) i d f(t)}{\|A\|\|B\|}
$$

SimRank: Jeh and Widom [Jeh and Widom 2002] realized that a more general way to attack the node similarity problem was to not only look for shared neighbors, that is, neighbors that are identical, but to look for neighbors that are similar. This produces the recursive statement, "Two objects are similar if they are related to similar objects" [Jeh and Widom 2002] Formally, their SimRank measure is defined as follows:

$$
\operatorname{sim}_{s r}(a, b)=\frac{c}{|I(a)||I(b)|} \sum_{x \in I(a)} \sum_{y \in I(b)} \operatorname{sim}_{s r}(x, y)
$$

if $a \neq b$. If $a=b$, then $\operatorname{sim}_{s r}(a, b)=1 . c$ is a constant $0<c<1$. Also, for SimRank and all its variants, if either $a$ or $b$ has no neighbors, then $\operatorname{sim}(a, b)=0$. SimRank can be computed iteratively by initializing the matrix of $\operatorname{sim}$ (.) values, hereafter called the $S$ matrix, to the identity matrix. A SimRank similarity value can be interpreted as the probability that two simultanous random walkers, starting at $a$ and $b$, will eventually meet, if they traverse the network backwards along directed edges. At each step, there is a probability $(1-c)$ that one or both walkers will abandon the walk and exit the network.

Obviously, we can add the effects of in-neighbors and out-neighbors to produce a more comprehensive measure of the neighbor similarity between two objects. Several authors have proposed this [Lin et al. 2007; Zhao et al. 2009].

SimRank can be described as a recursive extension of the co-citation index. An important difference between the non-iterative algorithms in Section 2.3.2 and SimRank is that the earlier algorithms can be computed locally with a minimum of computational effort. With SimRank, however, to compute the similarity of even a single pair of objects, one has to consider the entire graph. This increases the computational requirements by a factor of $n^{2} k$, where $k$ is the number of iterations. Consequently, several authors [Lizorkin et al. 2008; Jia et al. 2009; Cai et al. 2009; Li et al. 2009] have worked to reduce both the computational and memory requirements for SimRank, for general and specific applications.

In addition to concerns about the computational efficiency of the original SimRank formula, there are some structural flaws which mar its elegance. First, SimRank scores sometimes 
decrease when we would intuitively expect them to increase. Suppose we have an objectpair that has all neighbors in common. Then $\operatorname{sim}_{s r}(a, b)=c / d, d$ is the degree of $a$ or $b$. As $d$ increases, this should mean stronger ties between $a$ and $b$, but clearly $\operatorname{sim}_{s r}$ actually decreases.

SimRank++: Antonellis et al. [Antonellis et al. 2008] partially compensates for this unwanted decrease by inserting an evidence factor. The more neighbors in common, the higher the evidence of similarity. They define evidence as

$$
e v(a, b)=\sum_{i=1}^{|N(a) \cap N(b)|} \frac{1}{2^{i}}, \quad \text { (7) }
$$

where $N(a)$ is the undirected neighbor set of $a$. If $a$ and $b$ have only one neighbor in common, $e v=1 / 2$. As the number of neighbors increases, $e v \rightarrow 1$. This yields the following similarity definition:

$$
\operatorname{sim}_{e v}(a, b)=e v(a, b) \cdot c \sum_{x=1}^{N(a)} \sum_{y=1}^{N(b)} \operatorname{sim}_{e v}(x, y)
$$

The very narrow range $[0.5,1]$ of the evidence factor, however, leads to the problem that $\operatorname{sim}_{e v}$ (.) values are no longer bounded to a maximum of 1 or even to a constant. Instead, the maximum depends on the maximum value of $\|N(a)\| \cdot\|N(b)\|$ for the graph. The authors make one more extension to support edge-weighted graphs. Their final measure is called SimRank++:

$$
\operatorname{sim}_{s p p}(a, b)=e v(a, b) \cdot c \sum_{x=1}^{N(a)} \sum_{y=1}^{N(b)} w_{a b} w_{b y} \operatorname{sim}_{s p p}(x, y)
$$

PSimRank: Fogaras and Rácz [Fogaras and Rácz 2005] realize that the cause of improper weighted of neighbor-matching in SimRank is due to the paired-random walk model. Ignoring the decay constant $c$ for the moment, SimRank values are equal to the probability that two simultaneous random walkers, starting at nodes $a$ and $b$, will eventually encounter each other. Even in the best case scenario, in which $a$ and $b$ have all the same neighbors in common, so that $N(a)=N(b)$, the probability that the two walkers will happen to choose the same neighbor is $1 / d_{a}$, which decreases as the degree increases. To emend this situation, Fogaras and Rácz introduce coupled random walks. They partition the event space into three cases:

1.

$$
\text { Probability } P_{1}=P(\text { a and } b \text { step to the same node })=\frac{|I(a) \cap I(b)|}{|I(a) \cap I(b)|}
$$

2.

$$
\text { Probability } P_{2}=P(\text { asteps toanode } \operatorname{in} I(a) \backslash I(b))=\frac{|I(a) \backslash I(b)|}{|I(a) \cup I(b)|}
$$

3.

$$
\text { Probability } P_{3}=P(\text { steps toanode } \operatorname{in} I(b) \backslash I(a))=\frac{|I(b) \backslash I(a)|}{|I(a) \cup I(b)|}
$$


Note that in case 1, which we would consider the direct similarity of $a$ and $b$, is described by the Jaccard Coefficient. As required, the sum of these probabilities equals 1 . We can then compute a similarity measure which takes the general form

$$
\operatorname{sim}_{p s}(a, b)=\sum_{i=1}^{3} P_{i} \cdot \operatorname{sim}(\text { neighbors in Case } i) .
$$

Noting that there are $\mid I(a) \backslash((b)|\cdot| I(b) \mid$ neighbor-pairs in Case 2 and $|I(b) \backslash \bigvee(a)| \cdot|I(a)|$ in Case 3 , this produces the logical but somewhat unwieldy formula:

$$
\operatorname{sim}_{p s}(a, b)=c\left[P_{1} \cdot 1+\frac{P_{2}}{|I(a) \backslash I(b)||I(b)|} \sum_{\substack{x \in I(a) \backslash I(b) \\ y \in I(b)}} \operatorname{sim}_{p s}(x, y)+\frac{P_{3}}{|I(b) \backslash I(a)||I(a)|} \sum_{\substack{x^{\prime} \in I(b) \backslash I(a) \\ y^{\prime} \in I(a)}} \operatorname{sim}_{p s}\left(x^{\prime}, y^{\prime}\right)\right] .
$$

MatchSim: The authors of MatchSim [Lin et al. 2009] take this emendment of random walking to its limit. They observe that when a human compares the features of two objects, a human does not select random features to see if they match. Rather, people look to see if there exists an alignment of features that produces a perfect or near-perfect matching. Therefore, their similarity measure discards the idea of random walk and replaces it with "the average similarity of the maximal matching between their neighbors" [Lin et al. 2009]:

$$
\operatorname{sim}_{m s}(a, b)=\frac{\sum_{(x, y) \in m_{a b}^{\star}} \operatorname{sim}_{m s}(x, y)}{\max (|I(a)|,|I(b)|)},
$$

where $m^{\star}$ represents the maximal matching. MatchSim omits the usual decay factor $c$, but this seems to be an idealization rather than a necessary alteration. Note that the size of the maximal matching is $\min (|I(a)|,|I(b)|)$. Without loss of generality, assume $a$ has fewer neighbors than $b$. The upper bound for $\operatorname{sim}_{m s}(a, b)$ occurs when every neighbor of $a$ is also a neighbor of $b$. In this special case,

$\max \left(\operatorname{sim}_{m s}(a, b)\right)=\max \left(\frac{\min (|I(a)|,|I(b)|)}{\max (|I(a)|,|I(b)|)}\right)=\frac{|I(a) \cap I(b)|}{|I(a) \cup I(b)|}$, which is the Jaccard coefficient.

PageSim: All of the previous works are modifications of the original SimRank measure and principles. We now consider two measures that are markedly different than SimRank. We first consider PageSim [Lin et al. 2006], which not only borrows the entire PageRank computation as a starting point, but also borrows the meaning of PageRank's iterative computation to devise a related computation. The canonical interpretation of PageRank is that for each step, each page sends out an equal fraction of its own importance to each of its neighbors. Its importance for the next step is the sum of the fractional importance it received from its in-neighbors. PageSim also uses this spreading or propagating mechanism; however, rather than there being a universal importance feature which can be summed, each node begins with a distinct self-feature, which is orthogonal to every other node feature. The authors describe the propagation process as occurring over distinct paths, and they sum the contributions of each path to compute the total distribution. As long as we permit self- 
intersecting paths, this is equivalent to measuring the random walk destination distribution for each node after $k$ steps. PageSim follows a multi-step procedure:

1. For each node $a$, define feature vector $F V(a) . F V_{b}(a)$ is the $b^{\text {th }}$ element of $F V(a)$.

2. Initialize all vectors: $F V_{a}^{0}(a)=\operatorname{PageRank}(a) \cdot F V_{b}^{0}(a)=0, b \neq a$.

3.

$$
\text { For } t=1 \text { to } k \text { iterations, } F V^{t}=c \cdot \sum_{a \in V} \frac{F V^{t-1}(a)}{|O(a)|}
$$

4. Measure the similarity between pairs of feature vectors. In their original paper [Lin et al. 2006], the similarity measure is defined thus:

$$
\operatorname{sim}_{p g 1}(a, b)=\sum_{i=1}^{n} \frac{\min \left(F V_{i}(a), F V_{i}(b)\right)^{2}}{\max \left(F V_{i}(a), F V_{i}(b)\right)}
$$

In an expanded work [Lin et al. 2007], they modify the formula to more closely resemble the Jaccard coefficient:

$$
\operatorname{sim}_{p g 2}(a, b)=\frac{\sum_{i=1}^{n} \min \left(F V_{i}(a), F V_{i}(b)\right)}{\sum_{i=1}^{n} \max \left(F V_{i}(a), F V_{i}(b)\right)}
$$

Leicht's Vertex Similarity: The last measure that we consider addresses the other major weakness of SimRank: it considers only equal-length paths of similarity. As stated earlier, a SimRank value equals the probability that a given pair of nodes will meet if they take steps simultaneously with the other. That is, it would not count a case where Walker $a$ takes 3 steps to reach $c$, and Walker $b$ takes 4 steps to reach $c$. To address this limitation, Leicht $e t$ al. [Leicht et al. 2005] formulate their measure from the following maxim:"Vertex $a$ is similar to $b$ if $a$ has any neighbor $c$ this is itself similar to $b$." On one hand, this statement explicitly supports asymmetrical pairs of paths. On the other hand, it assumes that being neighbors implies similarity. In Leichts model, it follows that neighbors are somewhat similar, which describes clustering rather than role classification.

The authors did not give a catchy or convenient name to their measure, so for convenience we will call it VertexSim (notated $\operatorname{sim}_{v}$ or $S_{v}$ ). The initial version of VertexSim, written in matrix form is

$$
\mathbf{S}_{\mathbf{v}}=\phi \mathbf{A} \mathbf{S}_{\mathbf{v}}+\mathbf{I}
$$

where $A$ is the adjacency matrix and $\varphi$ is a parameter to be determined. Solving for $\mathbf{S}_{\mathbf{v}}$ and performing a power series expansion, we get

$$
\mathbf{S}_{\mathbf{v}}=\mathbf{I}+\phi \mathbf{A}+\phi^{2} \mathbf{A}^{2}+\cdots
$$

After normalizing for the expected number of paths from $a$ to $b$ and some simplifying approximations, they authors finally derive the following: 


$$
\mathbf{S}_{\mathbf{v}}=\mathbf{D}^{-1}\left(\mathbf{I}-\frac{\mathbf{c}}{\lambda_{1}} \mathbf{A}\right)^{-1} \mathbf{D}^{-1}
$$

where $\lambda_{1}$ is the largest eigenvalue of $A$, and $D$ is the degree matrix ( $d_{i i}=$ degree of node $i$; all other $d_{i j}=0$ ). Here we have a closed form solution, which seems convenient, but we also need to invert two matrices. An iterative computation process being simpler, the authors rewrite the equation this way:

$$
\mathbf{D S}_{\mathbf{v}} \mathbf{D}=\frac{\mathbf{c}}{\lambda_{1}} \mathbf{A}\left(\mathbf{D S}_{\mathbf{v}} \mathbf{D}\right)+\mathbf{I}
$$

which we see resembles Eq. 14. The authors claim $\mathbf{D S}_{\mathbf{v}} \mathbf{D}$ can be initialized to any values such as $\mathbf{0}$ and will converge after 100 iterations or fewer.

We summarize these structural similarity measures in Table II

\section{AXIOMATIC ROLE SIMILARITY}

An equivalence relation is like a simple true-false indicator: it tells us nothing about degree of similarity. The real-world need is for a measure that not only recognizes automorphic equivalence, such as Smith child/spouse/parent to Jones child/spouse/parent (Figure 1), but also tells us that a Lee child is strongly similar to a Smith or Jones child, but not as similar to a Smith or Jones parent.

To deal with this shortcoming and to clarify the problem, we first identify a list of axiomatic properties that all role similarity measures should obey.

Definition 2. (Axiomatic Role Similarity Properties) Given a graph $G=(V, E)$, any $\operatorname{sim}(a$, b) that measures the neighbor-based role similarity between vertices $a$ and $b$ in $V$ should satisfy properties P1 to P5:

- $\quad$ P1) Range: $0 \leq \operatorname{sim}(a, b) \leq 1$, for all $a$ and $b$.

- P2) Symmetry: $\operatorname{sim}(a, b)=\operatorname{sim}(b, a)$.

- P3) Automorphism confirmation: If $a \equiv b, \operatorname{sim}(a, b)=1$.

- P4) Transitive similarity: If $a \equiv b$, then $\operatorname{sim}(a, c)=\operatorname{sim}(b, c)$.

- P5) Triangle inequality: $d(a, c) \leq d(a, b)+d(b, c)$, where distance $d(a, c)$ is defined as $1-\operatorname{sim}(a, c)$.

Any node similarity measure satisfying the first four conditions (without triangle inequality) is called an admissible role similarity measure. Any node similarity measure satisfying all five conditions is an admissible role similarity metric.

Some of these properties are representative of any node similarity measure, but Property 3 is an essential criterion which distinguishes a role similarity measure from other measures.

Property 1 describes the standard normalization where 1 means fully similar and 0 means completely dissimilar (i.e., the two neighborhoods have nothing in common). That is, we 
should always be able to recognize a purportedly equivalent node-pair by their similarity score of 1 . Property 2 indicates that similarity, like distance, must be symmetric. Property 3 expresses the requirement that if any nodes are automorphically equivalent, they must have full similarity to one another. As we discussed earlier, other definitions of role similarity are possible based on exact coloration or regular equivalence. Property 4 claims that the similarity between $c$ and $a$ is equal to the similarity between $c$ and any node equivalent to $a$. In other words, we can define the similarity between equivalence classes or orbits: $\operatorname{sim}(\Delta(u)$, $\Delta(v))=\operatorname{sim}(u, v)$. This guarantees consistency of values at an orbit-level. If Property 5 holds, the measure is metric-like, i.e., it satisfies the triangle inequality.

The triangle inequality requirement is much stronger than transitivity, enforcing an ordering of values. In a pure metric space, the distance between any two distinct items cannot be zero. Since our automorphic equivalence property allows equivalent items to have zero distance between them, our axioms define a pseudometric space.

Interestingly, Property 5 (triangle inequality) implies Property 4 (transitive similarity), which we prove below. Since not all similarity measures satisfy the triangle inequality, we specify Property 4 separately.

Corollary 1. If Axioms P1, P2, P3, and P5 hold, then P4 holds.

Proof: Let $a$ and $b$ be equivalent nodes. From the triangle inequality, we have $d(a, c) \leq d(a$, $b)+d(b, c) \leq d(b, c)$ because $d(a, b)=0$. Likewise $d(b, c) \leq d(b, a)+d(a, c) \leq d(a, c)$. The only way to reconcile $d(a, c) \leq d(b, c)$ and $d(b, c) \leq d(a, c)$ is if $d(a, c)=d(b, c)$.

The following corollary emphasizes a fundamental difference between role similarity measures and proximity-based similarity measures: role similarity does not decrease merely because distance increases.

Corollary 2. (Distance Independence): For every finite $k$, there exists a graph and a pair of nodes $a$ and $b$ such that the distance between $a$ and $b$ is at least $k$, and $\operatorname{sim}(a, b)=1$

Proof: Let $G(V, E)$ be a linear path graph with $n$ edges and $n+1$ vertices, where $n \geq k$. We label the vertices so that $V=\left\{v_{0}, v_{1}, \ldots, v_{n}\right\}$, and the edge set $E=\left\{\left(v_{0}, v_{1}\right),\left(v_{1}, v_{2}\right), \ldots\right.$, $\left.\left(v_{n-1}, v_{n}\right)\right\}$. It is clear that nodes that are the same distance from the endpoints are automorphically equivalent and thus role-equivalent. That is, $v_{j} \equiv v_{n-j}$, for all $0 \leq j \leq\lfloor n / 2\rfloor$.

We make a final observation. Our axiomatic role similarity model can just as easily find similarities between two or more graphs, because automorphism can be extended beyond single graphs. Graph isomorphism (an equivalence relation between two graphs) is a special case of graph automorphism (an equivalence relation within a single graph) in which we declare that the two graphs are indeed a single graph that is not connected. This distance independence is absent from prioximity-based node similarity measures. 


\subsection{Binary-Valued and Real-Valued Role Similarity Measures}

Every equivalence relation $\sigma$ has a corresponding binary-valued indicator function: $I_{\sigma}(u, v)$ $=1$ iff $u \equiv v$. Otherwise, $I_{\sigma}(u, v)=0$. This indicator function is a admissible axiomatic role similarity metric.

Theorem 1. (Binary Admissibility) Given any equivalence relation that also satisfies automorphism confirmation (P3), its binary indicator function is an admissible similarity metric.

Proof: Binary values satisfy the Range requirement (P1). Any equivalence relation satisfies symmetry (P2) and transitivity (P4), by definition. We prove that this indicator function satisfies the triangle inequality(P5), namely, $d(a, c) \leq d(a, b)+d(b, c)$, where $d(a, b)=1-$ $\operatorname{sim}(a, b)$, by considering all possible class assignments for $a, b$, and $c$ :

\begin{tabular}{llll}
\hline Case & Description & Distances & Tri. Inequality \\
\hline 1 & All in the same class & $d(a, c)=d(a, b)=d(b, c)=0$ & $0 \leq 0+0$ \\
2 & All in different classes & $d(a, c)=d(a, b)=d(b, c)=1$ & $1 \leq 1+1$ \\
3 & $a$ and $c$ in the same class & $d(a, c)=0$ & $0 \leq 1+1$ \\
4 & $b$ and one other in the same class & $d(a, b)=0$ or $d(b, c)=0$ & $1 \leq 0+1$ \\
\hline
\end{tabular}

We have shown that a binary indicator function for an equivalence relation satisfies properties $\mathrm{P} 1, \mathrm{P} 2, \mathrm{P} 4$, and $\mathrm{P} 5$. Thus, if we are given that $\mathrm{P} 3$ is also met, then all properties are met.

Note that automorphic equivalence, regular equivalence, and exact coloration all satisfy P3, so they are admissible metrics. Though these binary-valued similarity measures are admissible, they do not help us to understand the degree of similarity or dissimilarity. We would like a real-valued measure that ranks the degree of role similarity.

However, from the above discussion, we can see that the basic five axioms (P1-5) do not provide enough constraint for selecting real-valued role similarity. To achieve this we here introduce a basic property which can serve as a basic guideline for helping measure the degree of the role similarity. Note that this property is not an axiom, i.e., a real-valued role similarity may not meet its criterion. But it is intuitively appealing and can be a desirable supplement to help derive the real-valued role similarity.

Similarity Ordering: For any two vertices $a$ and $b$, let $d_{a}$ and $d_{b}$ be their degrees, respectively. Without loss of generality, let $d_{a}=\min \left\{d_{a}, d_{b}\right\}$ and $d_{b}=\max \left\{d_{a}, d_{b}\right\}$. Consider a hypothetical pair of vertices $\bar{a}$ and $b$ where $d_{\bar{a}}=d_{a}$ and $d_{b} \equiv d_{b}$. Moreover, every neighbor of $\bar{a}$ is also a neighbor of $b: \bar{N}(\bar{a}) \subseteq N(b)$. Given this, the role similarity of $a$ and $b$ should be no higher than the role similarity of $\bar{a}$ and $b$, i.e., $\operatorname{sim}(a, b) \preceq \operatorname{sim}(\bar{a}, b)$. In other words, $\bar{a}$ and $b$ serve as an upper bound benchmark to rank the role similarity. This is based on the insight that for any $d_{a}$ and $d_{b}, \bar{a}$ is the best possible replacement for $b \overline{\text { in terms of role }}$ (assuming each neighbor has equal importance). 
The similarity ordering criterion can be considered a supplement to Automorphism Confirmation (P3). It aims to deal with P3's limitation of only applying when two nodes are automorphic; $\mathrm{P} 3$ becomes silent when nodes are not equivalent. The criterion provides guidance for the many node-pairs which are not equivalent: In what way are they not equivalent, and how can we measure this easily? There are two ways to be different: having a different number of neighbors, or having neighbors which are not quite equivalent to one another. The similarity ordering bounds the similarity based on only the former, i.e., the difference between the number of neighbors. Note that here the underlying assumption is that each neighbor is considered to be an equal factor in measuring the role similarity. As our experimental results will show (Section 6), a real-valued role similarity measure satisfying this criterion (Section 4) produces superior role measure compared with the existing state-of-the-art approaches. (The alternative assumption which permits some neighbors to be more important can be a valid choice and may violate this criterion. However, it is beyond the scope of this paper and we leave it for the future work.)

In next section, we will leverage this criterion to develop a real-valued role similarity measure. Now, before presenting our proposed metric, we first examine some similarity measures proposed in earlier works.

\subsection{Similarity Measures That Are Not Axiomatically Admissible}

Table III categorizes the previous section's similarity measures with respect to key axiomatic role similarity properties: Automorphism Confirmation (P3) and Transitivity (P4). We also include the Similarity Ordering property. We omit range (P1) and symmetry (P2) axioms because they are not cause for rejecting any of these measures.

Bibliographic coupling and co-citation fail to meet the Automorphism Confirmation property because they only count similarity if nodes share the same neighbors. They require a path of length of 2 between the two nodes, so these measures do not satisfy the Distance Independence corollary.

Though SimRank and its variants seem to capture the intuition of recursive structural similarity, the random walk matching does not satisfy the basic graph automorphism condition. The SimRank similarity [Jeh and Widom 2002] between nodes $u$ and $v$ is based on the average similarity between $u$ 's neighbors and v's neighbors:

$$
\operatorname{sim}_{s r}(u, v)=\frac{(1-\beta)}{|N(u)||N(v)|} \sum_{x \in N(u)} \sum_{y \in N(v)} \operatorname{sim}_{s r}(x, y), \text { for } u \neq v, \operatorname{sim}_{s r}(v, v)=1,
$$

where $\beta$ is a decay factor, $0<\beta<1$, so that the influence of neighbors decreases with distance. The original SimRank measure is for directed graphs, but it works equally well for undirected graphs. SimRank values can be computed iteratively, with successively iterations approaching a unique solution, much as PageRank [Page et al. 1999] does.

SimRank is not an admissible role similarity measure-We give examples where property 3 (automorphism confirmation) does not hold. In Figure 3(a), $a$ and $b$ have the 
same neighbors. By even the strictest definition (structural equivalence), $a$ and $b$ have the same role. However, since SimRank's initial assumption is that there is no similarity among $c, d$, and $e$, when it computes the average similarity of $a$ and $b$ 's neighbors, it does not discover that $a$ and $b$ have equivalent neighborhoods. Assuming the best case where $c, d$, and $e$ are in fact equivalent and using $\beta=0.15, \operatorname{sim}_{s r}(a, b)$ converges to only 0.667 . Even if the neighbors are not equivalent to one another, $a$ to $b$ should still be equivalent, but SimRank will never discover this. We note that variants of SimRank [Antonellis et al. 2008; Fogaras and Rácz 2005; Li et al. 2009; Xi et al. 2005; Yin et al. 2006; Zhao et al. 2009] also do not meet the automorphic equivalence property for similar reasons.

MatchSim is not an admissible role similarity measure-MatchSim seems to solve SimRank's problem of using average neighbor similarity by using maximal matching instead. If two nodes have equivalent neighborhoods, then they will have a similarity score of 1. MatchSim still falls short, however, because its initial state is too pessimistic, and its iterations do not explore all possible equivalences. Initially, each node is equivalent to itself $\left(\operatorname{sim}^{0}(v, v)=1\right)$, but all distinct node-pairs are not $\left(\operatorname{sim}^{0}(u, v)=0\right.$ if $\left.u \neq v\right)$. With each iteration, it checks for similar neighbors. In the first iteration, it detects structural equivalence, identical to co-citation and bibliographic coupling. In the second iteration, it builds on the previous similarity scores and compares neighborhoods again. However, consider Figure 3(b) in which there is an odd distance between two nodes. Nodes $u$ and $v$ are automorphically equivalent, but because there are no nodes that are an equal distance from both $u$ and $v, \operatorname{sim}_{m s}(u, v)=0$ !

PageRank-based measures, such as PageSim, meet the automorphism and transitivity axioms, so they are axiomatically admissible. However, they do not satisfy the Similarity Ordering property. Sharing neighbors does not guarantee that similarity will be at least as good as not sharing neighbors. Consider Figure 3(c), where every neighbor of $\bar{a}$ is also a neighbor of $b$. However, their PageRank scores are quite different: $P R(\bar{a})=0.119$, but $P R(b)$ $=0.175$, using $\beta=0.9$. Vertex $a$ has the same degree as $\bar{a}$ and has no neighbors in common with $b$. Its PageRank score is 0.139 , so $\sin (a, b)>\operatorname{sim}(\bar{a}, b)$. We also note that in experimental evaluation (Subsection 6.6), PageSim performs poorly on measuring the realworld role similarity.

To our best knowledge, there is no available real-valued structural similarity measure satisfying the automorphic equivalence requirement and similarity ordering.

\section{ROLESIM: A REAL-VALUED ADMISSIBLE ROLE SIMILARITY}

To produce an admissible real-valued role similarity measure, we face two key challenges: First, it is computationally difficult to verify the automorphic equivalence property. Though not proven to be NP-complete, the graph automorphism problem has no known polynomial algorithm [Fortin 1996]. Second, all the existing real-valued role similarity measures have problems dealing with even simple conditions such as structural equivalence (Section 3.2). To meet these challenges, we take the following approach: Given an initial simplistic but admissible role similarity measurement for each pair of nodes, refine the measurement by expressing similarity in terms of neighboring values, while maintaining the automorphic and 
structural equivalence properties. Using this approach, we formally introduce RoleSim, the first admissible real-valued role similarity measure (metric) and its associated properties. It also satisfies the similarity ordering property.

\subsection{RoleSim Definition}

Given a graph $G=(V, E)$, the RoleSim measure realizes the recursive node structural similarity principle "two nodes are similar if they relate to similar objects" as follows.

Definition 3. (RoleSim metric) Given two vertices $u$ and $v$, where $N(u)$ and $N(v)$ denote their respective neighborhoods and $d_{u}$ and $d_{v}$ denote their respective degrees, then

$$
\operatorname{RoleSim}(u, v)=(1-\beta) \max _{M(u, v)} \frac{\sum_{(x, y) \in M(u, v)} \operatorname{RoleSim}(x, y)}{d_{u}+d_{v}-|M(u, v)|}+\beta
$$

where $x \in N(u), y \in N(v)$, and $M(u, v)$ is a matching between $N(u)$ and $N(v)$, i.e., $M(u, v)=$ $\left\{(x, y) \mid x \in N(u), y \in N(v)\right.$, and no other $\left(x^{\prime}, y^{\prime}\right) \in M(u, v)$, s.t., $x=x^{\prime}$ or $\left.y=y^{\prime}\right\}$. The parameter $\beta$ is a decay factor, $0<\beta<1$.

The decay factor, similar to the one used in PageRank [Page et al. 1999], both dampens the recursive effect and guarantees a minimal RoleSim score of $\beta$. We will sometimes abbreviate $\operatorname{RoleSim}(u, v)$ as $R(u, v)$. $\mathbf{R}$ refers to the entire matrix of values. Figure 4 illustrates the matching process. Vertex $u$ has three neighbors $\left(x_{1}, x_{2}, x_{3}\right)$, and $v$ has four neighbors $\left(y_{1}, y_{2}, y_{3}, y_{4}\right)$. The $(x, y)$ grid is the subset of the RoleSim matrix of values corresponding to the pairings of neighbors of these two vertices. A matching selects one cell per row and column. If the number of rows differs from the number of columns, then the matching size is limited to $|M(u, v)|=\min \left(d_{u}, d_{v}\right)$. A maximal matching is a matching where the total value of selected cells is maximum. In contrast, SimRank computes the average of every cell in the neighbor grid.

\subsubsection{Relation to Jaccard and Tanimoto Coefficients-RoleSim employs a} generalization of the Jaccard coefficient, which measures the commonality between two sets

$A$ and $B$ as $J(A, B)=\frac{|A \cap B|}{|A \cup B|}$. Previous works [Fogaras and Rácz 2005] have used this index to compare node neighborhoods; several variants exist [Melançon and Sallaberry 2008]. Our denominator is similar to that of the Tanimoto coefficient [Tanimoto 1958], which measures similarity between multisets or between vectors. In our generalization, however, sets $A$ and $B$ are not vectors and need not share any common elements; instead, there is a weighted matching $M$ between similar elements in $A$ and $B$, i.e., $(a, b) \in M, a \in A, b \in B$. Let $r(a, b)$ $\in[0,1]$ record the similarity between $a$ and $b$.

Definition 4. (Generalized Jaccard Coefficient) The generalized Jaccard coefficient measures the similarity between two sets $A$ and $B$ under matching $M$, defined as

$$
J(A, B \mid M)=\frac{\sum_{(a, b) \in M} r(a, b)}{|A|+|B|-|M|}
$$


The original Jaccard coefficient is a special case which uses the following matching $M$ : Let $r(x, y)=1$ if $x=y$; otherwise 0 . Then define $M=\{r(x, x) \mid x \in A, x \in B\}$. Thus, the generalized Jaccard coefficient $J(A, B \mid M)$ reduces to $J(A, B)$. Comparing Eq. (17) and (18), we see that the heart of $\operatorname{RoleSim}(u, v)$ is equivalent to the maximum of the generalized Jaccard coefficient between $N(u)$ and $N(v)$, among all matchings $M(u, v)$. Then,

$$
\operatorname{RoleSim}(u, v)=(1-\beta) \max _{M(u, v)} J(N(u), N(v) \mid M(u, v))+\beta
$$

4.1.2. Relation to Weighted Matching-The definition and significance of RoleSim( $u$, $v)$ is closely related to maximal weighted matching. In our case, the matching is between the neighboring nodes of $u$ and $v$.

\section{Definition 5. Maximal Neighborhood Matching Mu, v)}

Let $R(x, y)$ be a similarity score between any two nodes $x$ and $y$ ( 0 if no score exists). Given two nodes $u$ and $v$, their neighborhood matching $M(u, v)$ is a weighted bipartite matching between neighbor sets $N(u)$ and $N(v)$ where the weights are the $R(x, y)$ scores. The weight of the matching is $w(M)=\Sigma_{(x, y) \in M} R(x, y)$. A maximal matching $\mathbb{M} u$, v) is an $M$ with maximum weight.

Using this, we can represent $\operatorname{RoleSim}(u, v)$ in terms of maximal weighted matching $\mathbf{M}$ In Figure 4, the shaded cells represent the maximal matching: $0.7+0.6+0.3=1.6$.

Theorem 2. (Maximal Weighted Matching) The RoleSim between nodes $u$ and $v$ corresponds linearly to the maximal weighted matching $\mathbb{M}$ for the bipartite graph $(N(u)$, $N(v), N(u) \times N(v))$, with each edge $(x, y) \in N(u) \times N(v)$ having the weight $\operatorname{RoleSim}(x, y)$ :

$$
\operatorname{RoleSim}(u, v)=(1-\beta) \frac{w(\mathbb{M})}{\max \left(d_{u}, d_{v}\right)}+\beta
$$

Proof: We need to show that Equations (17) and (20) are equivalent. Without loss of generality, let $d_{u} \geq d_{v}$. First, we show that the cardinality of the maximal weighted matching $\mathbb{M}=\min \left(d_{u}, d_{v}\right)=d_{v}$. It cannot be greater, because there are insufficient elements in $d_{v}$. It cannot be smaller, because if it were, there would exist an available edge between an uncovered node in $d_{u}$ with one in $d_{v}$. Adding this edge would increase the matching (every edge has weight $\geq \beta$ ). If $\mid \mathbb{M}=\min \left(d_{u}, d_{v}\right)$, it follows that $d_{u}+d_{v}-|M|=\max \left(d_{u}, d_{v}\right)$. Thus, the denominators in Equations (17) and (20) are constant and identical. It is then a trivial observation that the numerators are in fact the same. Therefore, the maximal value for the entire Equation (17) is the same as the value in (20).

Theorem 2 not only shows the key equilibrium of role similarities between pairs of nodes in a graph $G$, but it also shows that RoleSim may be computed using existing maximal matching algorithms. Finally, given Theorem 2, we can state the following lemma:

Lemma 4.1. RoleSim satisfies the Similarity Ordering criterion. 
Proof: Consider any two vertices $a$ and $b$ where $a$ 's degree is not larger: $d_{a} \leq d_{b}$. Let $\bar{a}$ and $b$ be hypothetical vertices where $d_{\bar{a}}=d_{a}, d_{b} \equiv d_{b}$. Moreover, every neighbor of $\bar{a}$ is a neighbor

of $b:{ }^{-}(\bar{a}) \subseteq N(b)$. Due to this maximal sharing of neighbors, $\operatorname{RoleSim}(\bar{a}, \bar{b})=(1-\beta) \frac{d_{a}}{d_{b}}+\beta$.

In general, however, $w\left(\mathbb{M} \leq d_{a}\right.$. Thus, $\operatorname{RoleSim}(a, b) \leq(1-\beta) \frac{d_{a}}{d_{b}}+\beta=\operatorname{RoleSim}(\bar{a}, \bar{b})$.

\subsection{RoleSim Computation}

RoleSim values can be computed iteratively and can be guaranteed to converge, just as in PageRank and SimRank. First we outline the iterative procedure. In the next section, we prove that the calculated values comprise an admissible role similarity metric.

Step 1: Let the initial matrix of RoleSim scores be $\mathbf{R}^{\mathbf{0}}$, estimated but admissible scores between any pair of nodes in $G$. Simple initialization schemes are described in Section 4.4.

Step 2: Compute the $k^{\text {th }}$ iteration $\mathbf{R}^{\mathbf{k}}$ scores from the $(k-1)^{\text {th }}$ iteration's values, $\mathbf{R}^{\mathbf{k}-\mathbf{1}}$. Specifically, for any nodes $u$ and $v$,

$$
R^{k}(u, v)=(1-\beta) \max _{M(u, v)} \frac{\sum_{(x, y) \in M(u, v)} R^{k-1}(x, y)}{d_{u}+d_{v}-|M(u, v)|}+\beta
$$

Based on Theorem 2, we compute Equation (21) by finding the maximal weighted matching in the weighted bipartite graph $(N(u), N(v), N(u) \times N(v))$ with each edge $(x, y) \in N(u) \times N(v)$ having weight $\left.R^{k-1}(x, y)\right)$.

Step 3: Repeat Step 2 until $\left|\mathbf{R}^{\mathbf{k}}-\mathbf{R}^{\mathbf{k}-1}\right|<$ some threshold $\delta$ for each pair of nodes in $G$.

Theorem 3. (Guaranteed Termination) For any admissible set of initial RoleSim ${ }^{0}$ values and any termination threshold $\delta>0$, the change in RoleSim values between iterations will become arbitrarily small, e.g., for any $(u, v)$ pair,

$$
\lim _{k \rightarrow \infty} \mid \operatorname{RoleSim}^{k}(u, v)-\text { RoleSim }^{k-1}(u, v) \mid<\delta
$$

This can be proven by showing that the sequence of maximum absolute differences between any $\mathbf{R}^{\mathbf{k}}(u, v)$ and $\mathbf{R}^{\mathbf{k}+\mathbf{1}}(u, v)$, for $k=1,2, \ldots$, is a nonnegative geometric sequence monotonically decreasing and converging to 0 . The detailed proof is in Appendix A.

Unlike PageRank and SimRank which converge to values independent of the initialization, RoleSim values are sensitive to the initialization. Rather than being a disadvantage, this sensitivity provides the necessary relaxation to compute automorphic role similarity in polynomial time, by utilizing the initialization as prior knowledge.

\subsection{Admissibility of RoleSim}

Here, we present one of the key contributions of this article: the axiomatic admissibility of RoleSim. If the initial computation is admissible, and because the iterative computation of 
Equation (20) maintains admissibility (i.e., is an invariant transform of the axiomatic properties), then the final measure is admissible.

Theorem 4. (Invariant Transformation) If the $k^{\text {th }}$ iteration RoleSim $^{k}$ is an admissible role similarity metric, then so is RoleSim ${ }^{k+1}$.

For each axiomatic property $P$, we must show "If the $k^{\text {th }}$ iteration RoleSim $^{k}$ satisfies Axiom $P$, then so does RoleSim ${ }^{k+1}$." Properties 1 (Range) and 2 (Symmetry) are trivially invariant, so we will focus on the other three.

Automorphism Confirmation Invariance Proof: For nodes where $u \equiv v$, there is a permutation $\sigma$ of vertex set $V$, such that $\sigma(u)=v$, and any edge $(u, x) \in E$ iff $(v, \sigma(x)) \in E$. This indicates that $\sigma$ provides a one-to-one equivalence between nodes in $N(u)$ and $N(v)$. Also, $u$ and $v$ have the same number of neighbors, i.e., $d_{u}=d_{v}$. So, it is clear that the maximal weighted matching Min the bipartite graph $(N(u), N(v), N(u) \times N(v))$ selects $d_{u}=d_{v}$ pairs of weight 1 each. Thus,

$\operatorname{RoleSim}^{k+1}(u, v)=(1-\beta) \frac{w(\mathbb{M})}{\max \left(d_{u}, d_{v}\right)}+\beta=(1-\beta) \frac{d_{u} \cdot 1}{d_{u}}+\beta=1$.

Transitive Similarity Invariance Proof: Assume transitivity holds for iteration $k$ : for any $a$ $\equiv b, c \equiv d, \operatorname{RoleSim}^{k}(a, c)=\operatorname{RoleSim}^{k}(b, d)$. Denote the maximal weighted matching between $N(a)$ and $N(c)$ as $\mathrm{M}$ Since there is a one-to-one equivalence correspondence $\sigma$ between neighborhoods $N(a)$ and $N(b)$ and a one-to-one equivalence correspondence $\sigma^{\prime}$ between $N(c)$ and $N(d)$, we can construct a matching $\mathbb{M}$ between $N(b)$ and $N(d)$ as follows: $\mathbb{M}$ $=\left\{\left(\sigma(x), \sigma^{\prime}(y)\right) \mid(x, y) \in \mathbb{M}\right.$. Since transitive similarity holds for RoleSim ${ }^{k}$, we have $\operatorname{RoleSim}^{k}(x, y)=\operatorname{RoleSim}^{k}\left(\sigma(x), \sigma^{\prime}(y)\right)$. Thus, $w(\mathbb{M})=w(\mathbb{M}$, and

$$
(1-\beta) \frac{w(\mathbb{M})}{\max \left(d_{a}, d_{c}\right)}+\beta=(1-\beta) \frac{w\left(\mathbb{M}^{\prime}\right)}{\max \left(d_{b}, d_{d}\right)}+\beta \operatorname{RoleSim}^{k+1}(a, c)=\operatorname{RoleSim}^{k+1}(b, d) .
$$

Triangle Inequality Invariance Proof: For iteration $k$, for any nodes $a, b$, and $c, d^{k}(a, c) \leq$ $d^{k}(a, b)+d^{k}(b, c)$, where $d^{k}(a, b)=1-\operatorname{RoleSim}^{k}(a, b)$. We must prove that this inequality still holds for the next iteration: $d^{k+1}(a, c) \leq d^{\mathrm{k}+1}(a, b)+d^{k+1}(b, c)$.

Observation: if there is any matching $M$ between $N(a)$ and $N(c)$ which satisfies $1-\left((1-\beta) \frac{w(M)}{d_{c}}+\beta\right) \leq d^{k+1}(a, b)+d^{k+1}(b, c)$, then $d^{k+1}(a, c) \leq d^{k+1}(a, b)+d^{k+1}(b, c)$. This is because $\frac{w(M)}{d_{c}} \leq \frac{w(\mathbb{M})}{d_{c}}$, where Mis the maximal weighted matching between $N(a)$ and $N(c)$, and thus, $1-\left((1-\beta) \frac{w(M)}{d_{c}}+\beta\right) \geq 1-\left((1-\beta) \frac{w(\mathbb{M})}{d_{c}}+\beta\right)=d^{k+1}(a, c)$.

We break down the proof into three cases:

Case 1. $\left(d_{b} \leq d_{a} \leq d_{c}\right)$,

Case 2. $\left(d_{a} \leq d_{b} \leq d_{c}\right)$, and 
Case 3. $\left(d_{a} \leq d_{c} \leq d_{b}\right)$.

Case 1: Since $d_{b}$ is smallest, this sets the size for the maximal neighborhood matchings: $\mathbb{M} a$, $b)|=| \mathrm{M} b, c) \mid=d_{b}$. Define candidate matching $M$ between $N(a)$ and $N(c)$ as $M=\{(x, z) \mid(x, y)$ $\in \mathbb{M} a, b) \wedge(y, z) \in \mathbb{M} b, c)\}$. Then using our observation above:

$$
\begin{aligned}
d^{k+1}(a, b) & \\
+ & d^{k+1}(b, c) \\
- & \left(1-(1-\beta) \frac{w(M)}{d_{c}}\right. \\
& -\beta)=(1-\beta)[ \\
- & \frac{w(\mathbb{M}(a, b))}{d_{a}} \\
- & \frac{w(\mathbb{M}(b, c))}{d_{c}} \\
+ & \left.\frac{w(M)}{d_{c}}\right]+1-\beta=(1-\beta)\left[\frac{d_{b}-w(\mathbb{M}(a, b))}{d_{a}}\right. \\
- & \frac{d_{b}}{d_{a}}+\frac{d_{b}-w(\mathbb{M}(b, c))}{d_{c}} \\
- & \frac{d_{b}}{d_{c}}-\frac{d_{b}-w(M)}{d_{c}} \\
+ & \left.\frac{d_{b}}{d_{c}}\right]+1-\beta \geq(1-\beta)[1 \\
- & \frac{d_{b}}{d_{a}}+\frac{\sum_{(x, y) \in \mathbb{M}(a, b)}\left(1-R^{k}(x, y)\right)}{d_{c}} \\
+ & \frac{\sum_{(y, z) \in \mathbb{M}(b, c)}\left(1-R^{k}(y, z)\right)}{d_{c}} \\
& \left.-\frac{\sum_{(x, z) \in M}\left(1-R^{k}(x, z)\right)}{d_{c}}\right] \geq(1 \\
& -\beta)\left[\frac{\sum_{(x, y, z)}\left(d^{k}(x, y)+d^{k}(y, z)-d^{k}(x, z)\right)}{d_{c}}\right] \geq 0
\end{aligned}
$$

where $(x, y, z)$ means $(x, y) \in \mathbb{M} a, b),(y, z) \in \mathbb{M} b, c)$, and $(x, z) \in M$

Cases 2 and 3 can be proven by a similar technique; the complete proof is in Appendix A.

By combining the admissible initial configurations given in Sec 4.4 with Theorem 4 on invariance, we have shown that the iterative RoleSim computation generates a real-valued, admissible role similarity measure.

Theorem 5. (Admissibility) If the initial RoleSim ${ }^{0}$ is an admissible role similarity measure, then at each $k$-th iteration, RoleSim ${ }^{k}$ is also admissible. When RoleSim computation converges, the final measure lim $_{k \rightarrow \infty}$ RoleSim$^{k}$ is admissible. 


\subsection{Initialization}

According to Theorem 5, an initial admissible RoleSim measurement $\mathbf{R}^{\mathbf{0}}$ is needed to generate the desired real-valued role similarity ranking. What initial admissible measures or prior knowledge should we use? We consider three schemes:

1. $\mathbf{A L L - 1}: R^{0}(u, v)=1$ for all $u$, v.

2. Degree-Binary (DB): If two nodes have the same degree $\left(d_{u}=d_{v}\right)$, then $R^{0}(u, v)=$ 1 ; otherwise, 0 .

3.

$$
\text { Degree-Ratio (DR): } R^{0}(u, v)=(1-\beta) \frac{\min \left(d_{u}, d_{v}\right)}{\max \left(d_{u}, d_{v}\right)}+\beta \text {. }
$$

These schemes come from the following observation: nodes that are automorphically equivalent have the same degree. Equal degree is a necessary but not sufficient condition for automorphism. This observation is key to RoleSim: degree affects both the size of a maximal matching set and the denominator of the Jaccard Coefficient.

We make the following interesting observations about these initialization schemes.

Lemma 4.2. Let $\mathbf{R}^{\mathbf{1}}(A L L-1)$ be the matrix of RoleSim values at the first iteration after $\mathbf{R}^{\mathbf{0}}=$ $\mathbf{1}$ (All-1 initialization). Let $\mathbf{R}^{\mathbf{0}}(D R)$ be the matrix of RoleSim initialized by the Degree-Ratio $(D R)$ scheme. Then, $\mathbf{R}^{\mathbf{1}}(A L L-1)=\mathbf{R}^{\mathbf{0}}(D R)$.

This lemma can be easily derived by following the definition of RoleSim formula. Basically, the Degree-Ratio (DR) is exactly equal to the RoleSim state one iteration after ALL-1 initialization. Thus, ALL-1 and DR generate the same final results. The simple formula for DR is much faster than neighbor matching, so DR is essentially one iteration faster. On the other hand, we may consider the simple ALL-1 scheme to be sufficient, since it works as well as the more sophisticated DR. After the simple ALL-1 initialization, RoleSim's maximal matching process automatically discriminates between nodes of different degree and progressively learns the differences among neighbors as it iterates.

Theorem 6. (Admissible Initialization) ALL-1, Degree-Binary, and Degree-Ratio are all admissible role similarity metrics.

Proof: It is easy to see that ALL-1 degenerately satisfies all the axioms of a role similarity metric. For Degree-Ratio, Lemma 4.2 shows that its matrix of values is equivalent to ALL-1's matrix after one RoleSim iteration. Since RoleSim iterations will preserve the admissibility of a metric (Theorem 4), Degree-Ratio is also a metric. For Degree-Binary, it trivially satisfies Range(P1), Symmetry(P2), and Automorphism Confirmation (P3). For Transitive similarity (P4), we only need to show that $R^{0}(u, v)$ depends only on class membership. Class is defined by degree, and the measurement clearly depends only on degree. Finally, because Degree-Binary is a binary indicator of equivalence, Theorem 1 states that it is a role similarity metric.

Note that $\operatorname{SimRank}$ s and MatchSim's initialization $\left(\operatorname{sim}^{0}(u, v)=1\right.$ iff $\left.u=v\right)$ is NOT admissible, because it sets the initial value of any potentially equivalent node-pairs to 0 . 
SimRank and MatchSim iterations try to build up from zero. However, due to problems with structural equivalence and odd-length paths that we noted, they will never increase the value enough to discover all of the equivalent pairs that were neglected at the start.

In addition, both ALL-1 and DR initialization have the following convergence property, which is stronger than our earlier guaranteed termination property:

Theorem 7. (Monotone Convergence) If ALL-1 initialization is used, each RoleSim value is monotonically decreasing (or non-increasing): $\mathbf{R}^{\mathbf{k}+\mathbf{1}}(u, v) \leq \mathbf{R}^{\mathbf{k}}(u, v)$ for all $k$.

Proof: At any iteration, the RoleSim value for any $(u, v)$ is the maximal matching of its neighbors. The value can increase only if some neighbor matchings increase. If no value increased in the previous iteration, then no value can increase in the current iteration. In the first iteration after ALL-1, clearly no value increases. Therefore, no value ever increases. Any function that decreases monotonically and has a lower bound will converge. The lower bound value is $\beta$, so RoleSim with ALL-1 initialization is guaranteed to converge.

Indeed, this monotone convergence property can be generalized into the following format: if $\mathbf{R}^{\mathbf{1}} \leq \mathbf{R}^{\mathbf{0}}$ (that is, for every ( $u$, v) pair, $\mathbf{R}^{\mathbf{1}}(u, v) \leq \mathbf{R}^{\mathbf{0}}(u, v)$ ), then $\mathbf{R}^{\mathbf{k}+\mathbf{1}} \leq \mathbf{R}^{\mathbf{k}}$. Note that the Degree-Binary (DB) initialization scheme does not have this property. In our experiments, we make an empirical comparison of these initialization schemes.

\subsection{Computational Complexity}

Given $n$ nodes, we have $O\left(n^{2}\right)$ node-pair similarity values to update for each iteration. For each node-pair, we must perform a maximal weighted matching. For weighted bipartite graph $(N(u), N(v), N(u) \times N(v))$, the fastest algorithm based on augmenting paths (Hungarian method [Kuhn 1955]) can compute the maximal weighted matching in $O(x(x \log$ $x+y)$ ), where $x=|N(u)|+|N(v)|$ and $y=|N(u)| \times|N(v)|$.

A fast greedy algorithm offers a $\frac{1}{2}$-approximation of the globally optimal matching in $O(y$ $\log y)$ time [Avis 1983]. Furthermore, if an equivalence matching exists (i.e., $w(\mathbb{M}=\max$ $\left.\left(d_{u}, d_{v}\right)\right)$, the greedy method will find it. This is important, because it means that a greedy RoleSim computation still generates an admissible measure. Using greedy neighbor matching, the time complexity of RoleSim is $O\left(k n^{2} d^{\prime}\right)$, for $k$ iterations, where $d^{\prime}$ is the average of $y \log y$ over all vertex-pair bipartite graphs in $G$. The space complexity is $O\left(n^{2}\right)$. In the next section, we will introduce an approach for reducing both the time and memory cost.

\section{ICEBERG ROLESIM: A SCALABLE ALGORITHM}

Node similarity ranking in general is computationally expensive because we need to compute the similarity for $\left(\begin{array}{c}n \\ 2\end{array}\right)=O\left(n^{2}\right)$ node-pairs. A graph with 100, 000 nodes needs about 40GB memory to simply maintain the similarity values, assuming 8 bytes per value. Indeed, this is a major problem for almost all node similarity ranking algorithms. However, in most applications, we are interested only in the highest similarity pairs, which typically 
compose only a very small fraction of all pairs. Thus, in order to improve the scalability of RoleSim, we ask the following question: Can we identify the high-similarity pairs without computing all pair similarities? Formally, we consider the following question:

Definition 5.1. (Iceberg RoleSim) Given a threshold $\theta$, the Iceberg RoleSim problem is to discover all $(u, v)$ pairs for which $\operatorname{RoleSim}(u, v) \geq \theta$ and then approximate their RoleSim scores.

The goal is to identify and compute those high-similarity pairs without materializing the majority of the low similarity pairs. To solve Iceberg RoleSim, we consider a two-step approach: 1) use pruning rules to rule out pairs whose score must be less than $\theta$; and 2) apply RoleSim iterative computation to the remaining candidate pairs. Since RoleSim computation must match all neighbor-pairs $(N(u) \times N(v))$ of a candidate pair $(u, v)$, we have to handle neighbor-pairs (such as $x, y$ ) which are not themselves candidate pairs. Here, we employ upper and lower bounds for estimating RoleSim values for the non-candidate pairs.

\section{Upper and Lower Bound for RoleSim:}

Lemma 5.2. Given nodes $u$, $v$ and without loss of generality, $d_{u} \geq d_{v}$, if $d_{v} \leq \theta d_{u}$, then similarity $R(u, v) \leq(1-\beta) \theta+\beta$.

Proof: $R(u, v)=(1-\beta) \frac{w(\mathscr{M})}{d_{u}}+\beta \leq(1-\beta) \frac{d_{v}}{d_{u}}+\beta$

Given this, assuming $d_{u} \geq d_{v}$, since matching $0 \leq w(\mathscr{W}) \leq d_{v}$, then $R(u, v)$ is in the range $\left[\beta,(1-\beta) \frac{d_{v}}{d_{u}}+\beta\right]$. Furthermore, to facilitate our discussion, we further define $\theta^{\prime}=(\theta-\beta) /(1$ $-\beta)$. Now, we introduce the following pruning rules to filter out those pairs whose RoleSim cannot be greater than or equal to threshold $\theta$, without knowing their exact RoleSim scores (Without loss of generality, let $d_{u} \geq d_{v}$ ):

1. If $d_{v}<\theta^{\prime} d_{u}$, then $R(u, v)<\theta$

2. If maximal matching weight $w(\mathscr{M})<\theta^{\prime} d_{u}$, then $R(u, v)<\theta$

3. Assume neighbor lists $N(u)$ and $N(v)$ are sorted by degree, with $d_{1}^{u}$ and $d_{1}^{v}$ being the degrees of the first items. The maximum possible similarity of this pair is $m_{11}=(1-\beta) \frac{\min \left(d_{1}^{u}, d_{1}^{v}\right)}{\max \left(d_{1}^{u}, d_{1}^{v}\right)}+\beta$. If the shorter list has the smaller degree $\left(d_{1}^{v} \leq d_{1}^{u}\right)$, and if $m_{11}+d_{v}-1<\theta^{\prime} d_{u}$, then $R(u, v)<\theta$.

Rule 1 is just a restatement of Lemma 5.2. Rule 2 is based on the upper bound of RoleSim value. Rule 3 requires more explanation: continuing from Rule 2, we begin to consider all the pairings of neighbors. Because $d_{v}$ is the shorter list, every member must contribute to the final matching. Either $m_{11}$ will be in the matching or not. If it is, then an upper bound for $\mathscr{M}$ is if every remaining pair has weight 1 , yielding $m_{11}+\left(d_{v}-1\right)$. Additionally, because the lists are sorted, $d_{1}^{v} / d_{1}^{u} \geq d_{1}^{v} / d_{x}^{u}$, for $x>1$. So, if $m_{11}$ is too small to satisfy Rule 2 , then all pairings using $d_{1}^{v}$ are too small. This rule allows us to shortcircuit the full neighbor matching 


\subsection{Iceberg Algorithm}

We now outline our approach, which is formalized in Algorithm 1. To generate the initial iceberg hash map, we sort nodes by degree (line 3 ) and sort each node's list of neighbors, by degree (lines 4 to 6). The first sort allows us to consider only those node-pairs that are sufficiently similar in degree (line 8 , pruning rule 1 ). We compute the estimated similarity for the first pair of neighbors. Note that this estimatation formula is the same as DegreeRatio initialization. If this weight is below the limit defined in Rule 3, we terminate this pair's candidacy and move on (lines 9 to 12). Otherwise, compute the remainder of neighbor-pair initial similarities, and perform a maximal matching. If the matching weight exceeds the $\theta^{\prime}$ minimum bound (Rule 2), then this node-pair and its similarity are inserted into the hash table (lines 13 to 16). After iterating through all qualified node-pairs, we have our full hash table. We now perform RoleSim iterations, but only on members of the table, which is orders of magnitude smaller than a complete similarity matrix. When a noncandidate pair's value is needed (as a neighbor-pair of a candidate pair), we apply the following estimate based on its lower and upper bound (assuming $d_{u} \geq d_{v}$ ):

$$
\tilde{R}(u, v)=\alpha(1-\beta) \frac{d_{v}}{d_{u}}+\beta, \text { where } 0 \leq \alpha \leq 1 .
$$

In the experimental evaluation, we will empirical study the effect of $a$ on the estimation accuracy.

\subsection{Iceberg Computational Complexity}

Iceberg RoleSim's time and memory requirements are best understood as multiplicative improvement factors over standard RoleSim. For memory complexity, the factor is mainly the reduction in the number of stored node-pairs due to pruning. Let us define pruning factor $p$ as the ratio between the number of node-pairs stored in standard RoleSim and the number of top similarity values stored in an Iceberg hash table. Thus, $p=\left(\begin{array}{c}n \\ 2\end{array}\right) /|H| \approx \frac{n^{2}}{2|H|}$. Larger values mean a lower space requirement. Each hash table entry is a little more expensive than an equivalent similarity matrix entry, because it must store the vertex IDs $u$ and $v$ as well as $R(u, v)$. Consequently, the memory improvement factor is somewhat less than $p$.

The hash table size $|H|$ varies according to the graph's structural characteristics and the pruning threshold $\theta$. In our experiments with random power law graphs, we measured values of $p$ ranging from 33 to 814 . The largest reductions, by nearly 3 orders of magnitude, occur when higher values of $\theta$ are applied to graphs which are denser and larger, exactly when the improvement is needed most. This may be because these graphs have more internal structural diversity and thus less role similarity. 


\section{ALGORITHM 1}

$\operatorname{IcebergRoleSim}(G(V, E), \theta, \beta, \alpha)$

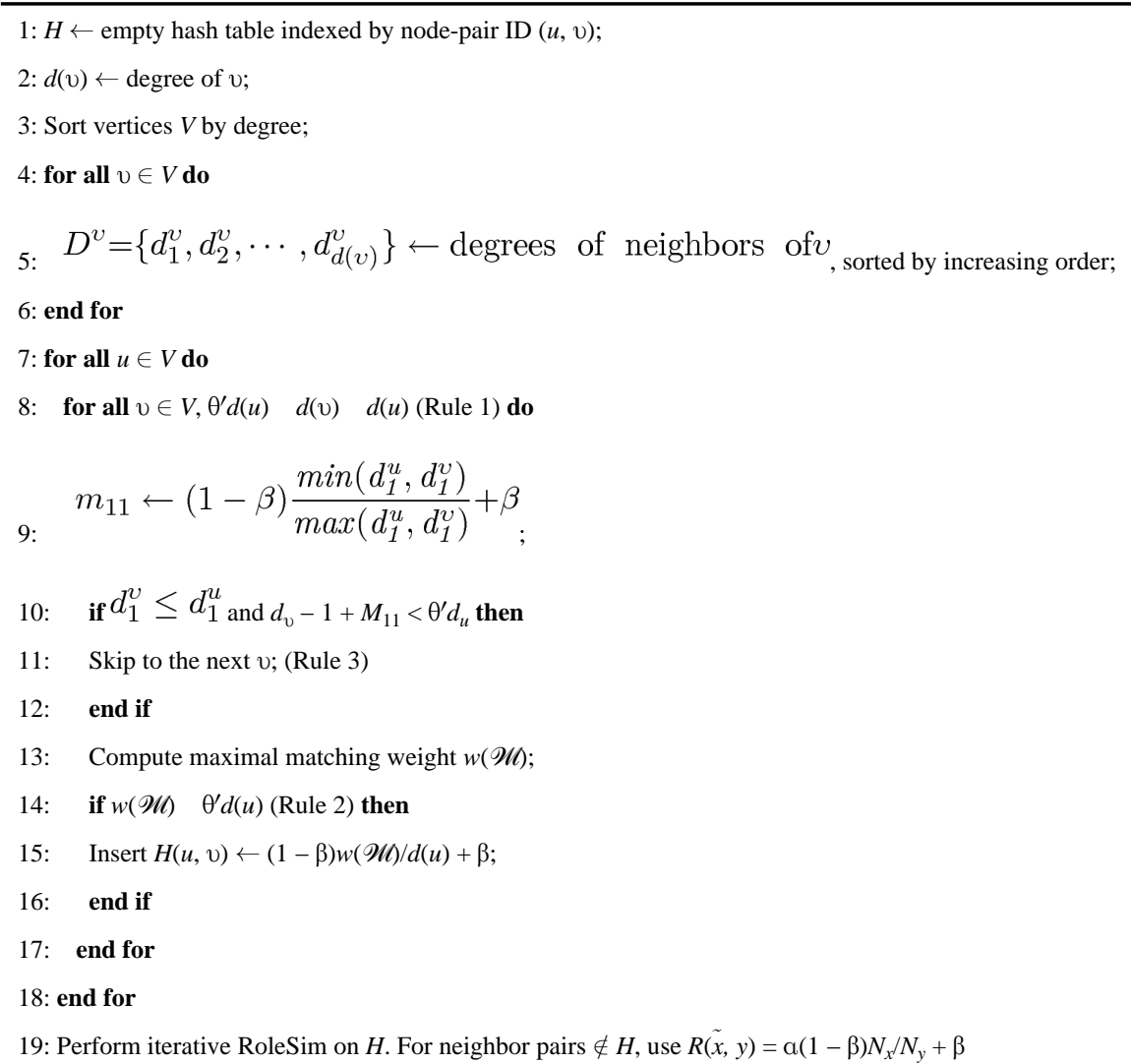

There are two major contributing factors to time complexity: the initial pruning and estimation of values, followed by the iterative refinement of values. The time complexity of the second stage (Line 19) is $p$ times faster than the time complexity of standard RoleSim, because Iceberg RoleSim iterates over the pruned hash tables instead of a complete $n \times n$ matrix. Moreover, the algorithm typically converges after fewer iterations, because there are fewer values being updated. Let us take a closer look at the initial stage, as detailed in Algorithm 1. Sorting the vertices by degree (Step 3 ) is a bucket sort, so it requires only $O(n)$ time. Sorting the neighbors of each node by degree (Steps $4-6)$ is $O(n \cdot d \hat{\log d} \hat{)}$, where $d \hat{=}$ expected (weighted average) node degree. Next comes the nested for loops (Lines 7 to 18), which implement a modified RoleSim iteration, incorporating pruning and estimation of values. Because the pruning is occuring amidst this loop, the time complexity falls in between that of a full standard RoleSim iteration and a $p$-pruned iteration. In practice, it is closer to a fully $p$-pruned iteration. So, the overall time complexity is $O\left(n \cdot d \hat{\log } d \hat{+} k^{\prime}|H| \underline{\mathrm{d}}\right.$ '), where $d^{\prime}$ is the same as in Section 4.5. 


\section{EXPERIMENTAL EVALUATION}

In this section we experimentally investigate the ranking ability and performance of the RoleSim algorithm for computing role similarity metric values. We compare RoleSim to several state-of-the-art node similarity algorithms, analyze the effect of different initialization schemes, and measure the scalability of Iceberg RoleSim. Specifically, we focus on the following questions:

1. How do different initialization schemes perform in terms of their final RoleSim score and computational efficiency?

2. Do node-pairs with high RoleSim scores actually have similar network roles? For any two nodes known to have similar network roles, do they receive high role similarity scores?

3. How much less memory and time does Iceberg RoleSim use, and how closely does its rankings match standard RoleSim's?

Clearly, the ideal validation study requires an explicit role model and role similarity measure, which often do not exist. In the following study, we utilize a well-known rolerelated random graph model and external measures of real datasets which provide strong role indication for these evaluations.

We set $\beta=0.1$ for both RoleSim and SimRank, defining convergence to be when values change by less than $1 \%$ of their previous values. We ran several RoleSim tests with both exact matching and greedy matching. The results were nearly identical (> $90 \%$ of cells have no difference; maximum difference was small), so we focus on greedy matching from here on. We implemented the algorithms in $\mathrm{C}++$. The large graph scalability tests were performed on Linux servers with $3.2 \mathrm{GHz}$ quad-core Xeon CPUs, $2 \mathrm{MB}$ cache, and $16 \mathrm{MB}$ RAM.

For our tests, we use three types of graphs:

- BL: the probabilistic block-model [Wang and Wong 1987], where each block is generally considered to be corresponding to a role [White et al. 1976]. Here, nodes are partitioned into blocks. Each node in block $i$ has probability $p_{i j}$ of linking to each node in block $j$. Thus, the underlying block-model may serve as the groundtruth for testing role similarity.

- SF: Large Scale-Free random graphs ${ }^{4}$ are used for testing scalability of the Iceberg RoleSim computation.

- Real-world networks, with a measureable feature similar to social role, are used for validating RoleSim performance.

$4_{\text {http://pywebgraph.sourceforge.net/ }}$ 


\subsection{Comparing Initialization}

In Section 4.4 we discussed that Degree-Ratio initialization generates the same results as ALL-1 by shortcutting the first iteration. This reduces the computation time by roughly $10 \%$. Now we ask: Does Degree-Binary initialization (DB, binary indicator which equals 1 when degrees $d_{u}=d_{v}$ ) give similar results, quickly?

We ran RoleSim using both ALL-1 and DB on 12 graphs, some scale-free and some blockmodel, having 500 to 10,000 nodes, and edge densities from 1 to 10 . We then converted values to percentile ranking, where $100 \%$ means the highest value and $50 \%$ is the median value. Test results are summarized in Table IV. The high correlation coefficient means the rankings are virtually identical, so the rankings are not very sensitive to the initialization method. Moreover, DB took 20\% from 68\% less time to converge. Overall, DB seems to be the preferred initialization scheme in terms of computational efficiency. Thus, we adopt it for the rest of the experiments.

\subsection{General Role Detection}

How well does RoleSim discover roles in complex graphs? Specifically, given a ground truth knowledge of roles, do nodes having similar roles have high scores? To answer this question, we generated probabilistic block-model graphs, where blocks behave like "noisy" roles, due to sampling variance. We generated graphs with $N=1000$ nodes and either 3 or 5 blocks. We varied the edge density $\frac{|E|}{|V|}$, with higher densities for graphs with more blocks. The size of each block and the $p_{i j}$ values were randomized; we generated 3 random instances for each graph class. We compared RoleSim to the state-of-the-art SimRank, SimRank++ [Antonellis et al. 2008], and P-SimRank [Fogaras and Rácz 2005].

For each measure and trial, we ranked the similarity scores. This serves to normalize the scoring among the four measures. Then, for each graph, we computed the average ranking of all pairs of nodes within the same block. We then averaged the three trials for each graph class.

Our results (Figure 5) show that RoleSim outperforms all other algorithms across all the tested conditions. None of the algorithms score perfectly, due to the inherent edge distribution variance of the probabilistic model. P-SimRank is better than SimRank, perhaps because it uses Jaccard Coefficient weighting, a step towards our RoleSim approach. Accuracy takes time. SimRank and SimRank++ run at the same speed. P-SimRank is about 1.5 to 2 times slower, and standard RoleSim is about twice as slow as SimRank.

\subsection{Real Dataset: Co-author Network}

Historically, structural role has not been measured precisely in large graphs, so it is difficult to find datasets with established ground-truth roles. In this experiment, we use a co-author nework and take author impact, as measured by G-index and H-index scores, as the ground truth. Based on recent studies, we expect network role in a co-author network to correlate well to author impact and thus serve as a predictive measure. Earlier investigations [Newman 2004; Otte and Rousseau 2002] observed structural patterns of collaboration in 
co-author networks and speculated that hubs (nodes with high degree) and connectors (nodes with high betweenness) would be likely candidates for high-impact authors. Recently, Yan and Ding [Yan and Ding 2009] discovered significant correlation between the number of citations and certain co-author network measures: betweenness centrality and PageRank similarity.

Our first dataset [Tang et al. 2008] is a co-author network of 2000 database researchers. Two authors are linked if they co-authored a paper from 2003 to 2008. We pruned the network to the largest connected component (1543 nodes, 15483 edges). An author's role depends recursively on the number of connections to other authors, and the roles of those others. Hence, it measures collaboration. We use the G-index as a proxy measure for co-author role (H-index provides similar results and thus is omitted here). The G-index measures the influence of a scientific author's publications, its value being the largest integer $G$ such that the $G$ most cited publications have at least $G^{2}$ citations. While G-index and co-author role are not precisely the same, G-index score is influenced strongly by the underlying role. High impact authors tend to be highly connected, especially with other high impact authors. If a paper is highly cited, this boosts the score of every co-author. Thus, we expect that if two authors have similar G-index scores, their node-pair is likely to have a high role simlarity value. To normalize RoleSim, P-SimRank, and G-index values, we converted each raw value to a percentile rank.

Figure 6(a) addresses our second validation question (high rank $\rightarrow$ similar roles?). For the top ranked $0.01 \%$ of author-pairs, their difference in G-index ranking is about 20 points, for both RoleSim and P-SimRank, well below the random-pair value of 33. A below-average difference confirms that the authors are relatively similar. However, as we expand the search towards $10 \%$, RoleSim continues to detect authors with similar authorship performance, while P-SimRank converges to random scoring.

To validate role $\rightarrow$ rank performance, we binned the authors into 10 roles based on G-index value (bottom $10 \%$, next $10 \%$, etc.). For every pair of authors within the same role decile, we looked up role similarity percentile rank and computed an average per bin. We also computed averages for pairs of authors not in the same bin (dissimilar roles). Figure 7 shows our results. The average within-bin RoleSim value is consistently between 55\% and 60\%, better than the random-pair score of 50, and independent of whether the G-index is high or low. It performs equally well for all roles. P-SimRank within-bin scores (dashed line), however, are inconsistent. Performance of P-SimRank is worse than random for low Gscores, perhaps due to low density of links in the network. For the cross-bin data, the X-axis is the difference in decile bins for the two authors in a pair. The falling line of RoleSim indicates that role similarity correctly decreases as G-index scores become less similar. For P-SimRank, however, the cross-bin scores (dashed line) hover around 50, equivalent to random scoring.

\subsection{Real Dataset: Internet Network}

Our second dataset is a snapshot of the Internet at the level of autonomous systems (22963 nodes and 48436 edges), as generated by [Newman 2006]. Several studies have confirmed that the Internet is hierarchically organized, with a densely connected core, medium density 
islands, a low density connecting mesh, and stubs (singly-connected nodes) at the periphery [Tauro et al. 2001; Carmi et al. 2007]. A node's position within the network (proximity to a hub or to a bridge between hubs) and its relation to others (such as density of connections) affects its efficiency for routing and its robustness. Proximity to the core is not a sufficient descriptor of role. For example, nodes which are in different local hubs might play the same role, even though they are far apart and may have somewhat different relationships to the main core. In [Carmi et al. 2007], $K$-shell decomposition is shown to be an effective way to partition the graph into its hierarchical components, because it addresses how many connections and to whom. We use RoleSim to partition the nodes into role classes and compare this to the $K$-shell partitions.

The $K$-core of a graph is the induced subgraph where every node connects to at least $K$ other nodes in the subgraph. If $K^{\prime}>K$, then the $K^{\prime}$-core must be an induced subgraph of the $K$ core. The $K$-shell is defined as the 'ring' of nodes that are included in a graph's $(K-1)$-core but not its $K$-core. In other words, we can decompose a graph into a set of nested rings, becoming denser as we move inward.

Using K-shells as our roles, we perform tests and analyses similar to those of the coauthor network. In Figure 6(b) we see that both measures do well for the top $0.1 \%$, but PSimRank's falters significantly when the range is expanded to the top $1 \%$.

Next, we treat $K$-shells the same way that we treated G-index decile bins in the previous test. See Figure 8. Unlike decile bins, the shells do not have equal sizes. K-shells 1, 2, and 3 together contain $92 \%$ of all nodes. To clarify how these three shells dominate, we also show horizontal lines representing the combined weighted average rank of all within-shell comparisons. RoleSim's within-shell values are consistently high, averaging $70 \%$. Conversely, P-SimRank finds strong above-average similarity for the small high-K shells, but nearly random similarity for shells 1 to 3 , pulling its overall performance down to $50 \%$.

In cross-shell analysis, RoleSim is able to distinguish different shells very well: RoleSim approaches zero as shell difference approaches maximum. On the other hand, P-SimRank shows almost no correlation to shell difference. Many of its scores are above-average when they should be below-average (dissimilar). On the whole, it seems that P-SimRank is not detecting role, but something related to connectedness and density.

In all these experiments, we can see that RoleSim provides positive answer to the role similarity ranking: 1) node-pairs with similar roles have higher RoleSim ranking than nodepairs with dissimilar roles, and 2) high RoleSim ranking indicates that nodes have similar roles. P-SimRank scores, however, do not correlate with network role similarity.

\subsection{Performance of Iceberg RoleSim}

In this experiment, we study how Iceberg RoleSim performs in terms of reducing computational time and storage, and its accuracy at approximating the RoleSim score for high similar node-pairs. Here, we generated 12 scale-free graphs with up to $100 \mathrm{~K}$ nodes and edge densities of 2 and 5, yielding up to $500 \mathrm{~K}$ edges. We compared standard RoleSim to Iceberg RoleSim, with $\theta$ values of 0.8 and 0.9 . The parameter $a$, which is the weighting for 
estimated non-stored values, is set to midpoint 0.5 . For the scale-free graphs, the relative scale of the iceberg compared to the full similarity matrix depends on $\theta$ and edge density, but it is almost independent of the number of nodes. Table V shows that the icebergs' hash tables are only $0.15 \%$ to $3.5 \%$ of the full similarity matrices. Higher density graphs tend to have more structural variation and thus fewer highly similar node pairs. In Figure 9, we see that Iceberg RoleSim is at least an order of magnitude faster. To check that the ranking has not changed significantly, we computed the Pearson correlation coefficient for each graph's Iceberg RoleSim's rankings vs. the rankings from the corresponding portion of the full similarity matrix. For $\theta=0.8$, the average coefficient is 0.823 , and for $\theta=0.9$, it is 0.880 . Both show very strong correlation, indicating Iceberg-RoleSim's very good accuracy at ranking role-similarity pairs.

Next we fixed $\theta$ at 0.9 and varied a from 0 to 1.0 to see how sensitive is the accuracy of Iceberg RoleSim with respect to a. The results from 6 scale-free graphs are shown in Figure 10. The labels describe the number of nodes and edges of each graph. Most graphs prefer a $=0$, but some prefer a midrange value. Any value in the lower half seems acceptable.

\subsection{Case Study: Coauthor Similarity}

To better illustrate the role similarity ranking ability of RoleSim we performed the following case study. We generated the coauthor network by taking all publications for SIGMOD, VLDB, ICDE, KDD, ICDM, and SDM from 2006 to 2011, as extracted from the DBLP database [Ley et al. 2012]. The resulting network contains 7072 nodes with 18994 edges. We computed the node similarity for all pairs of nodes using four algorithms (Iceberg RoleSim, SimRank, SimRank++, and PageSim). Then, for a given focal author, we found the 10 other authors that were most similar. We then used both G-index and H-index scores, obtained from the Microsoft Academic Search database [Microsoft Research 2012], as candidate measures of coauthorship role. If a particular similarity measure is a good role measure, then the 10 other authors should tend to have roles similar to that of the focal author, as measured by G- and H-indices. For example, we used Jaiwan Hei as our first focal author, who has a G-index score of 162. Our results are summarized in Table VI. Iceberg RoleSim found other high G-index authors, such as Philip Yu and Christos Faloutos. All 10 had high G-index and H-index scores. SimRank and SimRank++, on the other hand, found mostly low index authors. Since SimRank++ always performed better than SimRank, our tables show only the SimRank++ results. PageSim did a bit better. Its list includes a few strong authors, but most of the top 10 were also low G-index authors. We performed the same test for two other focal authors: Xifeng Yan, with G-index of 55 (Table VII), and Xiaolei Li, with G-index of 23 (Table VIII). Again, RoleSim was successful at finding authors with similar prestige, while the other algorithms were not.

\section{CONCLUSION}

We have developed RoleSim, the first real-valued role similarity measure that confirms automorphic equivalence. We have also presented a set of axioms which can test any future measure to see if it is an admissible measure or metric. Our experimental tests demonstrate RoleSim's correctness and usefulness on real world data, opening up exciting possibilities 
for scientific and business applications. At the same time, we see that other well-known measures, while suitable for other tasks, are not suitable for role similarity. This axiomatic approach may prove useful for developing and validating solutions to other related tasks.

\section{Acknowledgments}

This work was supported in part by the National Science Foundation under CAREER grant no. IIS-0953950, by the National Institute of General Medical Science of the National Institutes of Health (NIGMS/NIH) under Award no. R01GM103309 and by the Ohio Supercomputer Center under Grant no. PGS0218.

\section{APPENDIX}

\section{A. PROOFS OF THEOREMS AND LEMMAS}

Proof for Theorem 3 (Guaranteed Termination) Let $\delta^{k}(u, v)$ denote $\operatorname{RoleSim}^{k}(u, v)$ $\operatorname{RoleSim}^{k-1}(u, v)$, the difference of $\operatorname{RoleSim}(u, v)$ scores between iterations $k$ and $(k-1)$. Also, let $D_{k}=\max _{(u, v)}\left|\delta^{k}(u, v)\right|$ be the maximum absolute difference across all $u$ and $v$ in iteration $k$. For any node pair $(u, v)$, let the maximal weighted matching between $N(u)$ and $N(v)$ computed at iteration $k+1$ be $\mathscr{M}^{k+1}$. Note that its weight $w\left(\mathscr{M}^{k+1}\right)=\sum_{(x, y) \in \mathscr{M}^{k+1}}$ $\operatorname{RoleSim}^{k}(x, y)$, a summation of $|\mathscr{M}|=\min \left(d_{u}, d_{v}\right)$ terms. $|\mathscr{M}|$ is independent of $k$. Without loss of generality, assume $d_{u} \leq d_{v}$, so that $\max \left(d_{u}, d_{v}\right)=d_{v}$ and $|\mathscr{M}|=d_{u}$. Given this, we observe that

$w\left(\mathscr{M}^{k+1}\right)-\left(d_{v} \cdot D_{k}\right) \leq w\left(\mathscr{M}^{k+1}\right)-|\mathscr{M}| \cdot D_{k} \leq w\left(\mathscr{M}^{k}\right) \leq w\left(\mathscr{M}^{k+1}\right)+|\mathscr{M}| \cdot D_{k} \leq w\left(\mathscr{M}^{k+1}\right)+\left(d_{v} \cdot D_{k}\right)$

Therefore, $\left|w\left(\mathscr{W}^{k+1}\right)-w\left(\mathscr{W}^{k}\right)\right| \leq d_{v} \times D_{k}$. Then,

$$
\begin{aligned}
\mid \delta^{k+1} & (u, v) \mid \\
& =\mid \text { Rolesim }^{k+1}(u, v) \\
& - \text { Rolesim }^{k}(u, v) \mid \\
& =\mid(1-\beta) \frac{w\left(\mathscr{M}^{k+1}\right)}{d_{v}} \\
& -(1-\beta) \frac{w\left(\mathscr{M}^{k}\right)}{d_{v}} \mid \\
& =\frac{(1-\beta)}{d_{v}} \mid w\left(\mathscr{M}^{k+1}\right) \\
& -w\left(\mathscr{M}^{k}\right) \mid \leq \frac{(1-\beta)}{d_{v}} d_{v} \times D^{k}
\end{aligned}
$$

Then $D^{k+1}=\max _{(u, v)}\left|\delta^{k+1}(u, v)\right| \leq(1-\beta) D^{k}$, so $D^{k}$ decreases exponentially as $k$ increases. Thus $\lim _{k \rightarrow \infty} D_{k}=0$ and $\lim _{k \rightarrow \infty}\left|\operatorname{RoleSim}^{k}(u, v)-\operatorname{RoleSim}^{k-1}(u, v)\right|<\delta$, for any $\delta>0$.

Proof for Lemma 4.3 (Triangle Inequality Invariant) For iteration $k$, for any nodes $a, b$, and $c, d^{k}(a, c) \leq d^{k}(a, b)+d^{k}(b, c)$, where $d^{k}(a, b)=1-\operatorname{RoleSim}^{k}(a, b)$. We must prove that this inequality still holds for the next iteration: $d^{k+1}(a, c) \leq d^{k+1}(a, b)+d^{k+1}(b, c)$. To 
facilitate our discussion, we abbreviate $\operatorname{RoleSim}^{k}(u, v)$ as $r(u, v)$, and without loss of generality, let $N_{a} \leq N_{c}$.

We utilize the following observation: if there is a matching M between $N(a)$ and $N(c)$ which satisfies $1-\left((1-\beta) \frac{w(M)}{N_{c}}+\beta\right) \leq d^{k+1}(a, b)+d^{k+1}(b, c)$, then $d^{k+1}(a, c) \leq d^{k+1}(a, b)$ $+d^{k+1}(b, c)$. This is because $\frac{w(M)}{N_{c}} \leq \frac{w(\mathscr{M})}{N_{c}}$, where $\mathscr{M}$ is the maximal weighted matching between $N(a)$ and $N(c)$, and thus,

$1-\left((1-\beta) \frac{w(M)}{N_{c}}+\beta\right) \geq 1-\left((1-\beta) \frac{w(\mathscr{M})}{N_{c}}+\beta\right)=d^{k+1}(a, c)$.

In addition, we also denote the maximal weighted matching between $N(a)$ and $N(b)$ as $\mathscr{M}(a$, $b$ ), and the maximal weighed matching between $N(b)$ and $N(c)$ as $\mathscr{M}(b, c)$. Now, we consider three cases characterizing the relationship between $N(a), N(b)$, and $N(c)$.

Case $1\left(N_{b} \leq N_{a} \leq N_{c}\right)$ : In this case, we observe $|\mathscr{M}(a, b)|=|\mathscr{M}(b, c)|=N_{b}$. Given this, we consider the following matching $M$ between $N(a)$ and $N(c)$ :

$$
M=\{(x, z) \mid(x, y) \in \mathscr{M}(a, b) \wedge(y, z) \in \mathscr{M}(b, c)\},|M|=N_{b}
$$

Then, we have the following relationships: 


$$
\begin{aligned}
d^{k+1}(a, b) & \\
+ & d^{k+1}(b, c) \\
& -\left(1-(1-\beta) \frac{w(M)}{N_{c}}\right. \\
& -\beta)=(1-\beta)[ \\
& -\frac{w(\mathscr{M}(a, b))}{N_{a}} \\
& -\frac{w(\mathscr{M}(b, c))}{N_{c}} \\
+ & \left.\frac{w(M)}{N_{c}}\right]+1-\beta=(1-\beta)\left[\frac{N_{b}-w(\mathscr{M}(a, b))}{N_{a}}\right. \\
& -\frac{N_{b}}{N_{a}}+\frac{N_{b}-w(\mathscr{M}(b, c))}{N_{c}} \\
& -\frac{N_{b}}{N_{c}}-\frac{N_{b}-w(M)}{N_{c}} \\
+ & \left.\frac{N_{b}}{N_{c}}\right]+1-\beta \geq(1-\beta)[1 \\
& -\frac{N_{b}}{N_{a}}+\frac{\sum_{(x, y) \in \mathscr{M}(a, b)}(1-r(x, y))}{N_{c}} \\
+ & \frac{\sum_{(y, z) \in \mathscr{M}(b, c)}(1-r(y, z))}{N_{c}} \\
& \left.-\frac{\sum_{(x, z) \in M}(1-r(x, z))}{N_{c}}\right] \geq(1 \\
& -\beta)\left[\frac{\sum_{(x, y, z)}\left(d^{k}(x, y)+d^{k}(y, z)-d^{k}(x, z)\right)}{N_{c}}\right] \geq 0 \text { where }(x, y) \in \mathscr{M}(a, b),(y, z) \in \mathscr{M}(b, c),(x, z) \in M \\
& \\
&
\end{aligned}
$$

Case $2\left(N_{a} \leq N_{b} \leq N_{c}\right)$ : In this case, we observe $|\mathscr{M}(a, b)|=N_{a}$ and $|\mathscr{W}(b, c)|=N_{b}$. It follows that there is a subset $n(b)$ of $N(b)$ of size $N_{a}$ that participates in both $\mathscr{M}(a, b)$ and $\mathscr{M}(b, c): n(b)=\{y \mid(y, z) \in \mathscr{M}(b, c) \backslash\{(y, z) \mid \nexists(x, y) \in \mathscr{M}(a, b)\}\}$. Given this, we consider the following matching $M$ between $N(a)$ and $N(c)$ :

$$
M=\{(x, z) \mid(x, y) \in \mathscr{M}(a, b) \wedge(y, z) \in \mathscr{M}(b, c)\},
$$

$|M|=N_{a}$. Then, we have the following relationships: 


$$
\begin{aligned}
& d^{k+1}(a, b) \\
& +d^{k+1}(b, c) \\
& -\left(1-(1-\beta) \frac{w(M)}{n_{c}}\right. \\
& -\beta)=(1-\beta)[ \\
& -\frac{w(\mathscr{M}(a, b))}{n_{b}} \\
& -\frac{w(\mathscr{M}(b, c))}{n_{c}} \\
& \left.+\frac{w(M)}{n_{c}}\right]+1-\beta=(1-\beta)\left[\frac{n_{a}-w(\mathscr{M}(a, b))}{n_{b}}\right. \\
& -\frac{n_{a}}{n_{b}}+\frac{n_{a}-w(\mathscr{M}(b, c))}{n_{c}} \\
& -\frac{n_{a}}{n_{c}}-\frac{n_{a}-w(M)}{n_{c}} \\
& \left.+\frac{n_{a}}{n_{c}}\right]+1-\beta \geq(1 \\
& -\beta)\left[1-\frac{n_{a}}{n_{b}}+\frac{\sum_{(x, y) \in \mathscr{M}(a, b)}(1-r(x, y))}{n_{c}}+\frac{\sum_{(y, z) \in \mathscr{M}(b, c) \backslash\{(y, z) \mid \nexists(x, y) \in \mathscr{M}(a, b)\}}(1-r(y, z))}{n_{c}}-\frac{n_{b}-n_{a}}{n_{c}}-\frac{\sum_{(x, z) \in M}(1-r(x, z)}{n_{c}}\right. \\
& -\beta)\left[1-\frac{n_{a}}{n_{b}}-\frac{n_{b}-n_{a}}{n_{c}}+\frac{\sum_{(x, y, z)}\left(d^{k}(x, y)+d^{k}(y, z)-d^{k}(x, z)\right)}{n_{c}}\right] \operatorname{where}(x, y) \in \mathscr{M}(a, b),(y, z) \in \mathscr{M}(b, c),(x, z) \in M \geq(1 \\
& -\beta)[1 \\
& \left.-\frac{n_{a}}{n_{b}}-\frac{n_{b}}{n_{c}}+\frac{n_{a}}{n_{c}}\right]=(1-\beta) \frac{n_{b} n_{c}-n_{a} n_{c}-n_{b}^{2}+n_{a} n_{b}}{n_{b} n_{c}} \\
& =(1-\beta) \frac{\left(n_{b}-n_{a}\right)\left(n_{c}-n_{b}\right)}{n_{b} n_{c}} \geq 0
\end{aligned}
$$

Case $3\left(N_{a} \leq N_{c} \leq N_{b}\right)$ : In this case, we observe $|\mathscr{M}(a, b)|=N_{a}$ and $|\mathscr{M}(b, c)|=N_{c}$. Given this, we consider the following matching $M$ between $N(a)$ and $N(c)$ :

$$
M=\{(x, z) \mid(x, y) \in \mathscr{M}(a, b) \wedge(y, z) \in \mathscr{M}(b, c)\}
$$

In addition, we define:

$$
M_{1}=\{(x, y) \mid(x, y) \in \mathscr{M}(a, b) \wedge \nexists(y, z) \in \mathscr{M}(b, c)\} M_{2}=\{(y, z) \mid(y, z) \in \mathscr{M}(b, c) \wedge \nexists(x, y) \in \mathscr{M}(a, b)\}
$$

In other words, $M_{1} \subset \mathscr{M}(a, b)$ and $M_{2} \subset \mathscr{M}(b, c)$ do not link to each other using intermediate node $y \in N(b)$. We further denotes $m_{1}=\left|M_{1}\right|, m_{2}=\left|M_{2}\right|, m_{3}=|M|$. Note that $m_{1}$ $=N_{\mathrm{a}}-m_{3}, m_{2}=N_{c}-m_{3}$, and $N_{b} \geq m_{1}+m_{2}+m_{3}$.

Then, we have the following relationships: 


$$
\begin{aligned}
& d^{k+1}(a, b) \\
& +d^{k+1}(b, c) \\
& -\left(1-(1-\beta) \frac{w(M)}{N_{c}}\right. \\
& -\beta) \geq d^{k+1}(a, b) \\
& +d^{k+1}(b, c) \\
& -\left(1-(1-\beta) \frac{w(M)}{N_{b}}\right. \\
& -\beta) \geq 1 \\
& -\beta-(1-\beta)\left(\frac{w(\mathscr{M}(a, b))}{N_{b}}\right. \\
& +\frac{w(\mathscr{M}(b, c))}{N_{b}} \\
& \left.-\frac{w(M)}{N_{b}}\right)=(1-\beta)(1 \\
& +\frac{m_{3}-w(\mathscr{M}(a, b))}{N_{b}} \\
& -\frac{m_{3}}{N_{b}}+\frac{m_{3}-w(\mathscr{M}(b, c))}{N_{b}} \\
& -\frac{m_{3}}{N_{b}}-\frac{m_{3}-w(M)}{N_{b}} \\
& \left.+\frac{m_{3}}{N_{b}}\right) \geq(1 \\
& -\beta)(1 \\
& -\frac{m_{3}}{N_{b}}+\frac{\sum_{(x, y) \in \mathscr{M}(a, b) \backslash M_{1}}(1-r(x, y))}{N_{b}} \\
& -\frac{m_{1}}{N_{b}}+\frac{\sum_{(y, z) \in \mathscr{M}(b, c) \backslash M_{2}}(1-r(y, z))}{N_{b}} \\
& \left.-\frac{m_{2}}{N_{b}}-\frac{\sum_{(x, z) \in M}(1-r(x, z))}{N_{b}}\right) \geq(1 \\
& -\beta)(1 \\
& \left.-\frac{m_{3}}{N_{b}}-\frac{m_{1}}{N_{b}}-\frac{m_{2}}{N_{b}}+\frac{\sum_{(x, y, z)}\left(d^{k}(x, y)+d^{k}(y, z)-d^{k}(x, z)\right)}{N_{b}}\right) \geq((x, y) \in \mathscr{M}(a, b),(y, z) \in \mathscr{M}(b, c),(x, z) \in M)(1 \\
& -\beta)(1 \\
& \left.-\frac{m_{1}+m_{2}+m_{3}}{N_{b}}\right) \geq 0
\end{aligned}
$$

\section{REFERENCES}

Antonellis, Ioannis; Garcia-Molina, Hector; Chang, Chi-Chao. Simrank++: query rewriting through link analysis of the clickgraph. Proc. VLDB Endow. 2008; 1:408-421. 2008.

Avis D. A survey of heuristics for the weighted matching problem. Network. 1983; 13:475-493. 1983. 
Batagelj, Vladimir; Doreian, Patrick; Ferligoj, Anuška. An optimizational approach to regular equivalence. Social Networks. 1992; 14:121-135. 1992.

Borgatti, Stephen P.; Everett, Martin G. Notions of position in social network analysis. Sociological Methodology. 1992; 22:1-35. 1992.

Borgatti, Stephen P.; Everett, Martin G. Two algorithms for computing regular equivalence. Social Networks. 1993; 15:361-376. 1993.

Cai, Yuanzhe; Cong, Gao; Jia, Xu; Liu, Hongyan; He, Jun; Lu, Jiaheng; Du, Xiaoyong. Ninth IEEE Int. Conf. Data Mining (ICDM). IEEE Computer Society; 2009. Efficient Algorithm for Computing Link-Based Similarity in Real World Networks; p. 734-739.

Carmi, Shai; Havlin, Shlomo; Kirkpatrick, Scott; Shavitt, Yuval; Shir, Eran. A model of Internet topology using k-shell decomposition. Proc. Nat'l Academy of Sci. (PNAS). 2007 Jul 27.104:11150-11154. 2007.

Cvetkovíc, Dragos M.; Doob, Michael; Sachs, Horst. Spectra of Graphs: Theory and Applications, 3rd Revised and Enlarged Edition. Wiley; 1998.

Doreian, Patrick; Batagelj, Vladimir; Ferligoj, Anuška. Generalized blockmodeling. Vol. Vol. 25. Cambridge Univ Press; 2005.

Dragan, Natalia; Collard, Michael L.; Maletic, Jonathan I. IEEE Int. Conf. Software Maintenance (ICSM). IEEE; 2009. Using method stereotype distribution as a signature descriptor for software systems; p. 567-570.

Everett, Martin G.; Borgatti, Stephen P. Exact colorations of graphs and digraphs. Social Networks. 1996; 18:319-331. 1996.

Fogaras, Dániel; Rácz, Balázs. Proc. 14th Int. Conf. World Wide Web (WWW). ACM; 2005. Scaling link-based similarity search; p. 641-650.

Fortin, Scott. Technical Report TR 96-20. Edmonton, Alberta, Canada: Dept. Computer Science, Univ. of Alberta; 1996. The Graph Isomorphism Problem.

Freeman, Linton C. A set of measures of centrality based on betweenness. Sociometry. 1977; 40:3541. 1977.

Godsil, Chris; Royle, Gordon. Algebraic Graph Theory. Springer-Verlag; 2001.

Hafner-Burton, Emilie M.; Kahler, Miles; Montgomery, Alexander H. Network analysis for international relations. International Organization. 2009; 63:559-592. 2009.

Holme, Petter; Huss, Mikael. Role-similarity based functional prediction in networked systems: application to the yeast proteome. J. R. Soc. Interface. 2005; 2:327-333. 2005. [PubMed: 16849190]

Jeh, Glen; Widom, Jennifer. Proc. 8th ACM SIGKDD Int. Conf. Knowledge Discovery and Data Mining (KDD). ACM; 2002. SimRank: a measure of structural-context similarity; p. 538-543.

Jia, Xu; Cai, Yuanzhe; Liu, Hongyan; He, Jun; Du, Xiaoyong. Proc. 5th Int. Conf. Advanced Data Mining and Applications (ADMA). Berlin, Heidelberg: Springer-Verlag; 2009. Calculating Similarity Efficiently in a Small World; p. 175-187.DOI: http://dx.doi.org/ 10.1007/978-3-642-03348-3_19

Jin, Ruoming; Lee, Victor E.; Hong, Hui. KDD. ACM; 2011. Axiomatic ranking of network role similarity; p. 922-930.

Kessler MM. Bibliographic coupling between scientific papers. American Documentation. 1963; 14:10-25. 1963.

Kuhn HW. The Hungarian method for the assignment problem. Naval research logistics quarterly. 1955; 2:83-97. 1955.

Lee, Victor E.; Ruan, Ning; Jin, Ruoming; Aggarwal, Charu. Managing and Mining Graph Data. Vol. Chapter 10. Springer: A Survey of Algorithms for Dense Subgraph Discovery; 2010. p. 303-336.

Leicht EA, Holme Petter, Newman Mark E. J. Vertex similarity in networks. Phys. Rev. E. 2005 Feb. 73(Issue 2):026120. 2005.

Ley, Michael; Herbstritt, Marc; Ackermann, Marcel R.; Hoffmann, Oliver; Wagner, Michael; von Keutz, Stefanie; Hostert, Katharina; Holzträger, Doris. The DBLP Computer Science Bibliography. Schloss Dagstuhl - Leibniz-Zentrum für Informatik; 2012. http:// www.informatik.uni-trier.de/ ley/db/ 
Li, Pei; Cai, Yuanzhe; Liu, Hongyan; He, Jun; Du, Xiaoyong. Proc. 13th Pacific-Asia Conf. Advances in Knowledge Discovery and Data Mining (PAKDD). Berlin, Heidelberg: Springer-Verlag; 2009. Exploiting the Block Structure of Link Graph for Efficient Similarity Computation; p. 389-400.DOI:http://dx.doi.org/10.1007/978-3-642-01307-2_36

Lin, Zhenjiang; King, Irwin; Lyu, Michael R. Proc. IEEE/WIC/ACM Int'l Conf. Web Intelligence. IEEE Computer Society; 2006. PageSim: A Novel Link-Based Similarity Measure for the World Wide Web; p. 687-693.

Lin, Zhenjiang; Lyu, Michael R.; King, Irwin. Proc. IEEE/WIC/ACM Int'l Conf. Web Intelligence. IEEE Computer Society; 2007. Extending Link-based Algorithms for Similar Web Pages with Neighborhood Structure; p. 263-266.http://www.cse.cuhk.edu.hk/ king/PUB/WI2007_Lin.pdf

Lin, Zhenjiang; Lyu, Michael R.; King, Irwin. Proc. 18th ACM conf. Inform. and knowledge manage. (CIKM). ACM; 2009. MatchSim: a novel neighbor-based similarity measure with maximum neighborhood matching; p. 1613-1616.

Lizorkin, Dmitry; Velikhov, Pavel; Grinev, Maxim; Turdakov, Denis. Accuracy estimate and optimization techniques for SimRank computation. Proc. VLDB Endow. 2008; 1(Issue 1):422433. 2008. DOI:http://dx.doi.org/10.1145/1453856.1453904.

Lorrain FP, White HC. Structural equivalence of individuals in networks. J. Math. Sociology. 1971; 1:49-80. 1971.

Luczkovich JJ, Borgatti Stephen P, Johnson JC, Everett Martin G. Defining and Measuring Trophic Role Similarity in Food Webs Using Regular Coloration. J. Theoretical Biology. 2003; 220:303321. 2003.

MacArthur, Ben D.; Sánchez-García, Rubén J.; Anderson, James W. Note: Symmetry in complex networks. J. Discrete Applied Math. 2008; 156:3525-3531. 2008.

Marx, Maarten; Masuch, Michael. Regular equivalence and dynamic logic. Social Networks. 2003; 25:51-65. 2003.

McKay BD. Practical Graph Isomorphism. Congressus Numerantium. 1981; 30:45-87. 1981.

Melançon, Guy; Sallaberry, Arnaud. Proc. 12th Int. Conf. Inform. Visual. Washington, DC: IEEE Computer Society; 2008. Edge Metrics for Visual Graph Analytics: A Comparative Study; p. 610-615.DOI: http://dx.doi.org/10.1109/IV.2008.10

Microsoft Research. Microsoft Academic Search. 2012 2012. http://academic.research.microsoft.com/ RankList?entitytype $=2 \&$ topdomainid $=2 \&$ subdomainid $=7$.

Newman, Mark. Internet network. 2006 Jul. 2006. http://www-personal.umich.edu/ mejn/netdata/.

Newman, Mark EJ. Coauthorship networks and patterns of scientific collaboration. Proc. Nat'1 Academy of Sci. (PNAS). 2004; 101(Suppl 1):5200-5205. 2004.

Otte, Evelien; Rousseau, Ronald. Social network analysis: a powerful strategy, also for the information sciences. J. information Science. 2002; 28:441-453. 2002.

Page, Lawrence; Brin, Sergey; Motwani, Rajeev; Winograd, Terry. Technical Report 1999-66. Stanford InfoLab; 1999. The PageRank Citation Ranking: Bringing Order to the Web. http:// ilpubs.stanford.edu:8090/422/1/1999-66.pdf

Read, Ronald; Corneil, Derek. The graph isomorphism disease. J. Graph Theory. 1977; 1:339-363. 1977.

Schultz, Michael; Liberman, Mark. Proc.e DARPA Broadcast News Workshop. Morgan Kaufmann; 1999. Topic detection and tracking using idf-weighted cosine coefficient; p. 189-192.

Small, Henry. Co-citation in the scientific literature: A new measure of the relationship between two documents. J. Amer. Soc. Information Sci. 1973; 24:265-269. 1973.

Sparrow, Malcolm K. A linear algorithm for computing automorphic equivalence classes: the numerical signatures approach. Social Networks. 1993; 15:151-170. 1993. DOI: http://dx.doi.org/ 10.1016/0378-8733(93)90003-4.

Tang, Jie; Zhang, Jing; Yao, Limin; Li, Juanzi. Proc. 17th Int. conf. World Wide Web (WWW). ACM; 2008. Extraction and mining of an academic social network; p. 1193-1194.DOI: http://dx.doi.org/ $10.1145 / 1367497.1367722$

Tanimoto TT. An elementary mathematical theory of classification and prediction. IBM Taxonomy Application M. and A.6. 1958 Nov.3 1958. 
Tauro, Sudhir L.; Siganos, Georgos; Palmer, C.; Faloutsos, Michalis. Proc. IEEE Global Telecomm. Conf. IEEE; 2001. A simple conceptual model for the internet topology; p. 1667-1671.

Wang, Yuchung J.; Wong, George Y. Stochastic blockmodels for directed graphs. J. American Statistical Assoc. 1987; 82:8-19. 1987. 397.

Wasserman, Stanley; Faust, Katherine. Social network analysis: methods and applications. Cambridge University Press; 1994.

White, Douglas R.; Reitz, Karl P. Graph and semigroup homomorphisms on networks of relations. Social Networks. 1983; 5:193-234. 1983.

White, Harrison; Boorman, Scott; Breiger, Ronald. Social structure from multiple networks. I: Blockmodels of roles and positions. Am. J. Sociology. 1976; 81:730-780. 1976.

Xi, Wensi; Fox, Edward A.; Fan, Weiguo; Zhang, Benyu; Chen, Zheng; Yan, Jun; Zhuang, Dong. Proc. 28th Int. ACM SIG Conf. Research and Develop. in Inform. Retrieval (SIGIR). ACM; 2005. SimFusion: measuring similarity using unified relationship matrix; p. 130-137.

Yan, Erjia; Ding, Ying. Applying centrality measures to impact analysis: A coauthorship network analysis. J. Am. Soc. Information Sci. and Technology. 2009; 60:2107-2118. 2009.

Yin, Xiaoxin; Han, Jiawei; Yu, Philip S. In Proc. 32nd Int. Conf. Very Large Data Bases (VLDB). VLDB Endowment; 2006. LinkClus: efficient clustering via heterogeneous semantic links; p. 427-438.

Zhao, Peixiang; Han, Jiawei; Sun, Yizhou. Proc. 18th ACM conf. Inform. and knowledge manage. (CIKM). New York, NY: ACM; 2009. P-Rank: a comprehensive structural similarity measure over information networks; p. 553-562. 


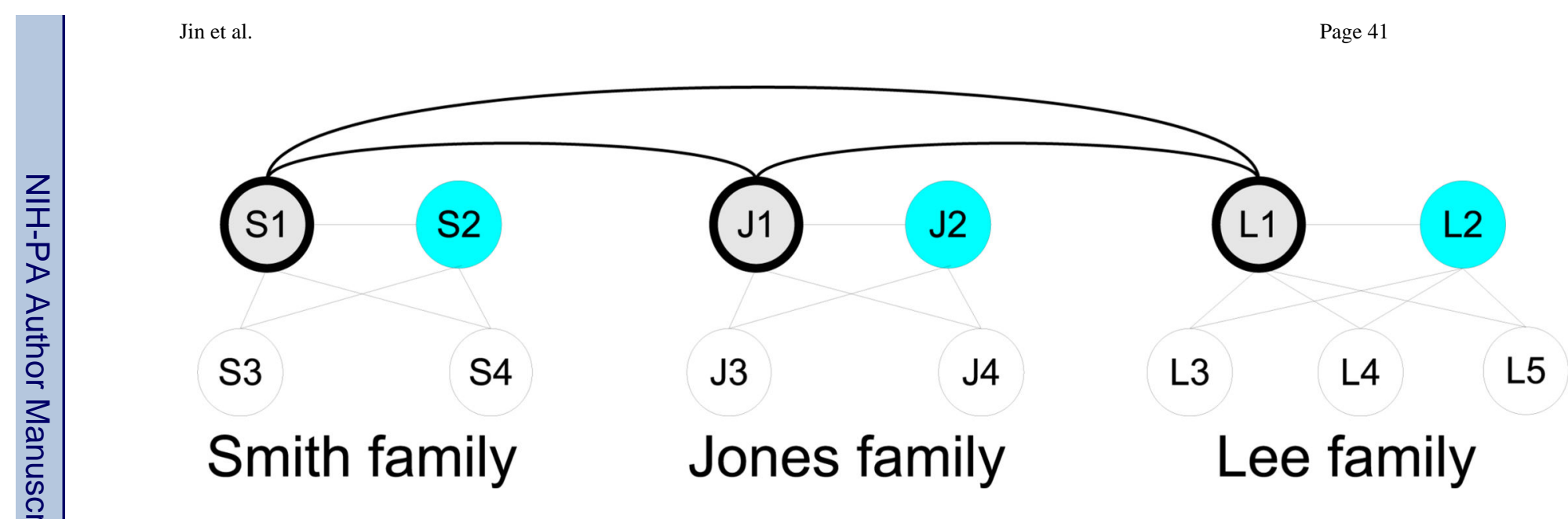

Fig. 1.

Example Graph for Role Equivalence 


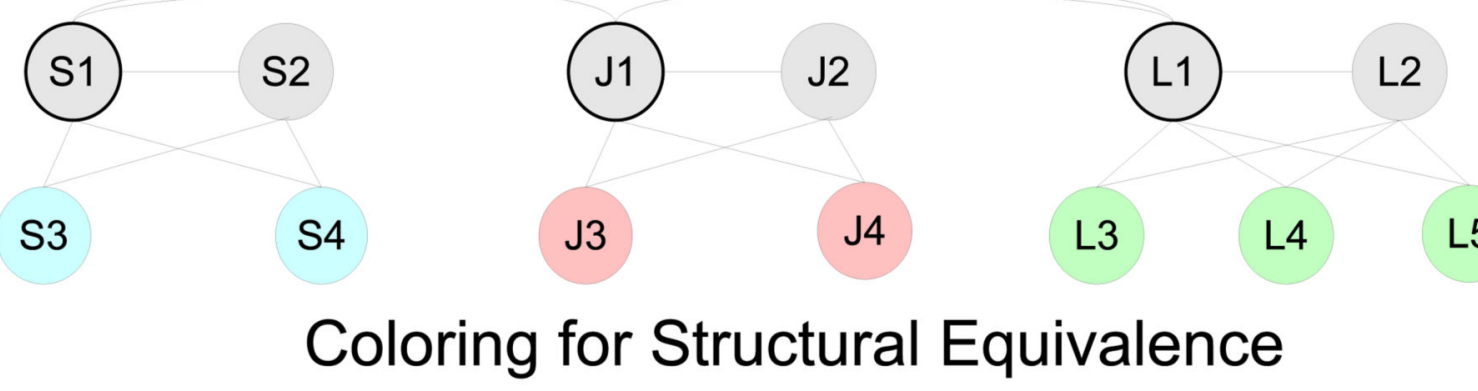

(a) Structural equivalence

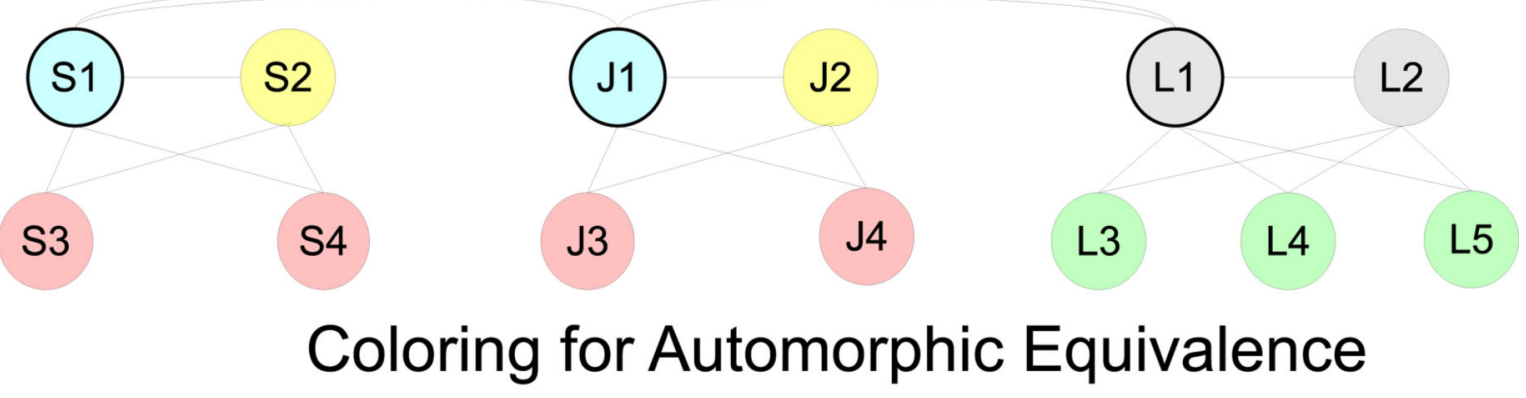

(b) Automorphic equivalence

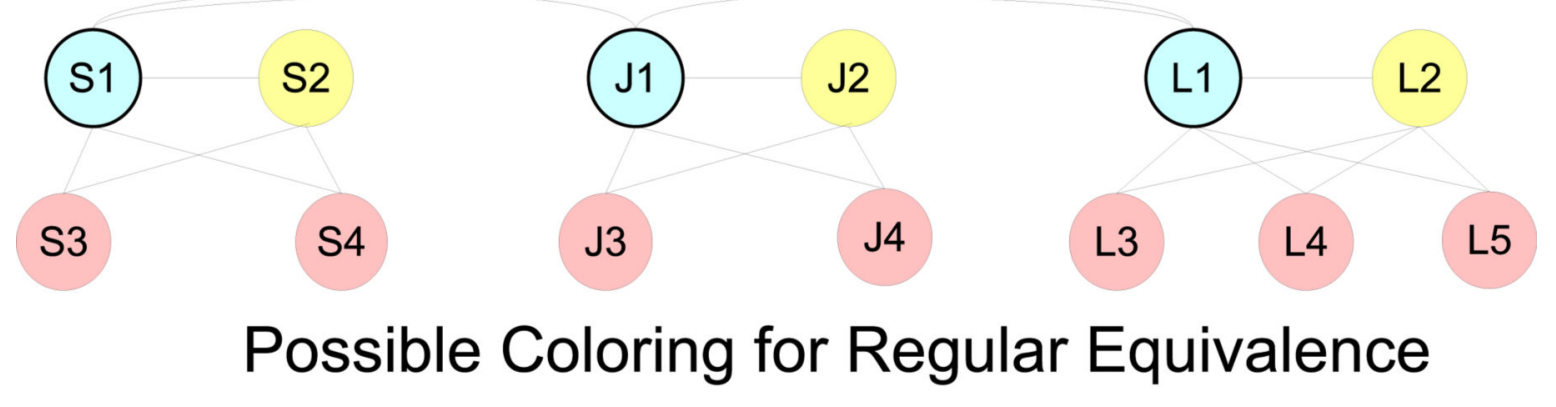

(c) Regular equivalence

Fig. 2.

Comparing Equivalence Schemes (Gray Nodes Are Not Equivalent) 
a

C

d e

b
W

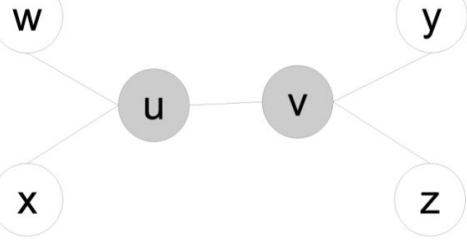

(b) Odd Distance

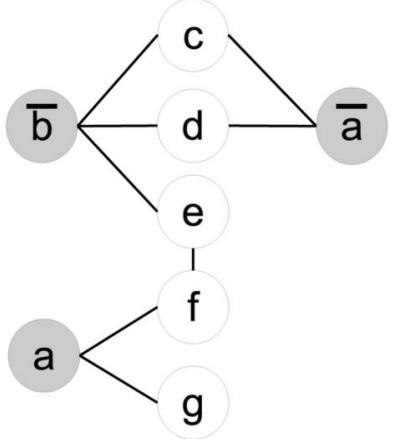

(c) Similarity Ordering (a) Structural equivalence

Fig. 3.

Problematic Configurations for SimRank, MatchSim, and PageSim 


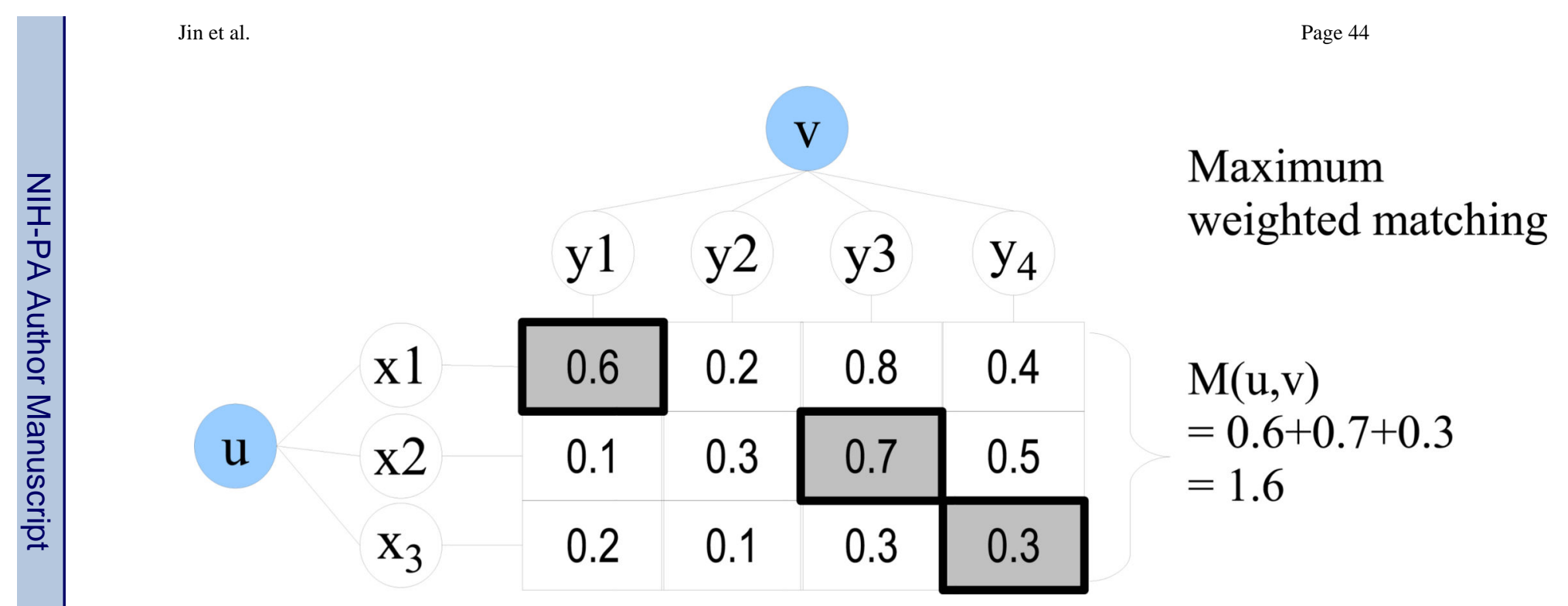

Fig. 4.

RoleSim(u,v) Based on Similarity of Their Neighbors 


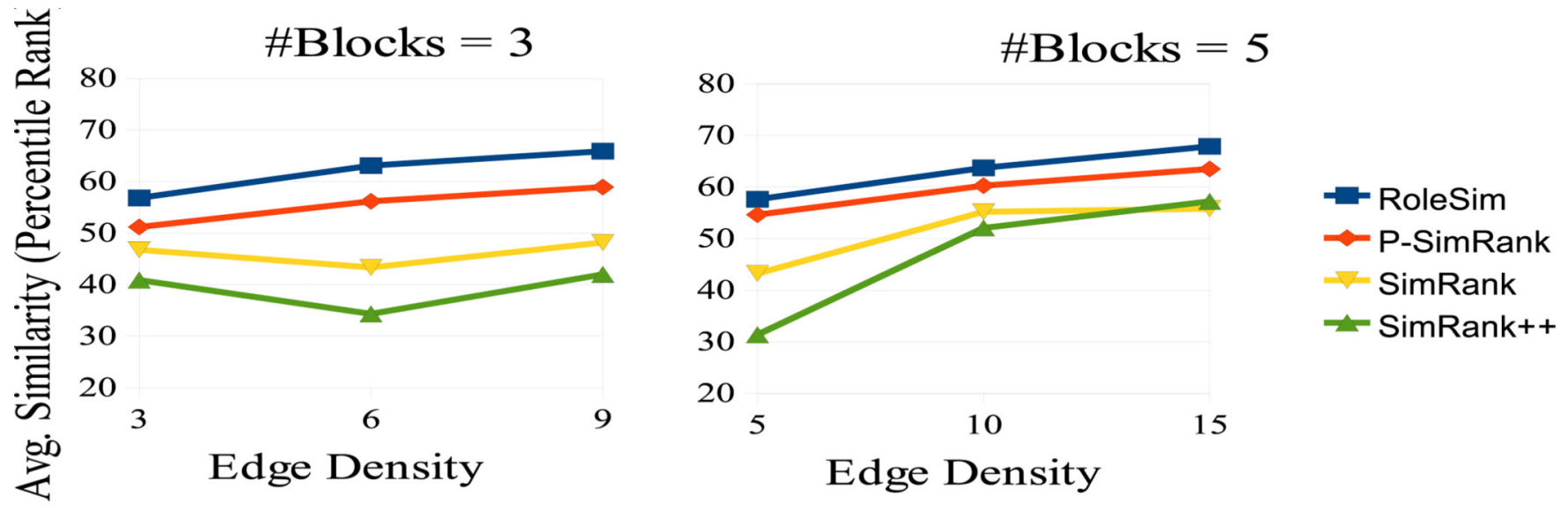

Fig. 5.

Avg. similarity ranking for nodes in the same block 


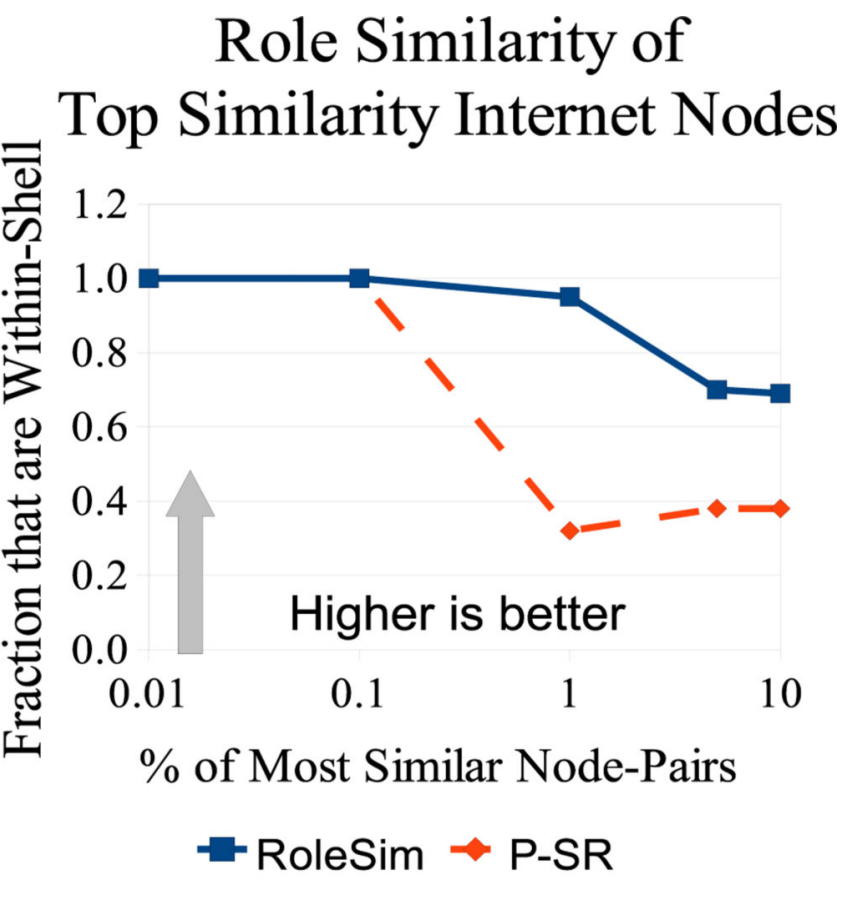

(a) Top Coauthors

(b) Top Internet nodes

Fig. 6.

Similarity of Nodes for Top Ranked Node-Pairs 


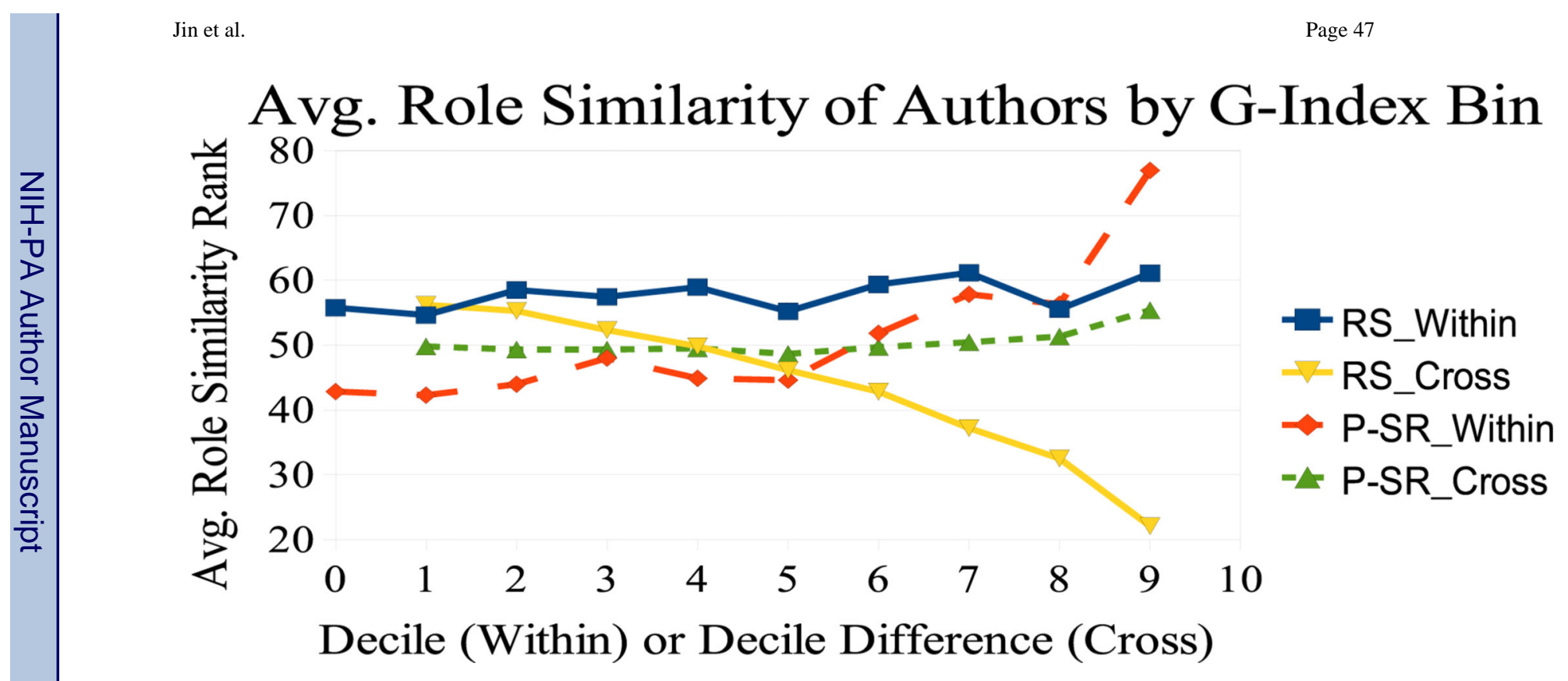

Fig. 7.

Similarity of Authors Binned by K-index 

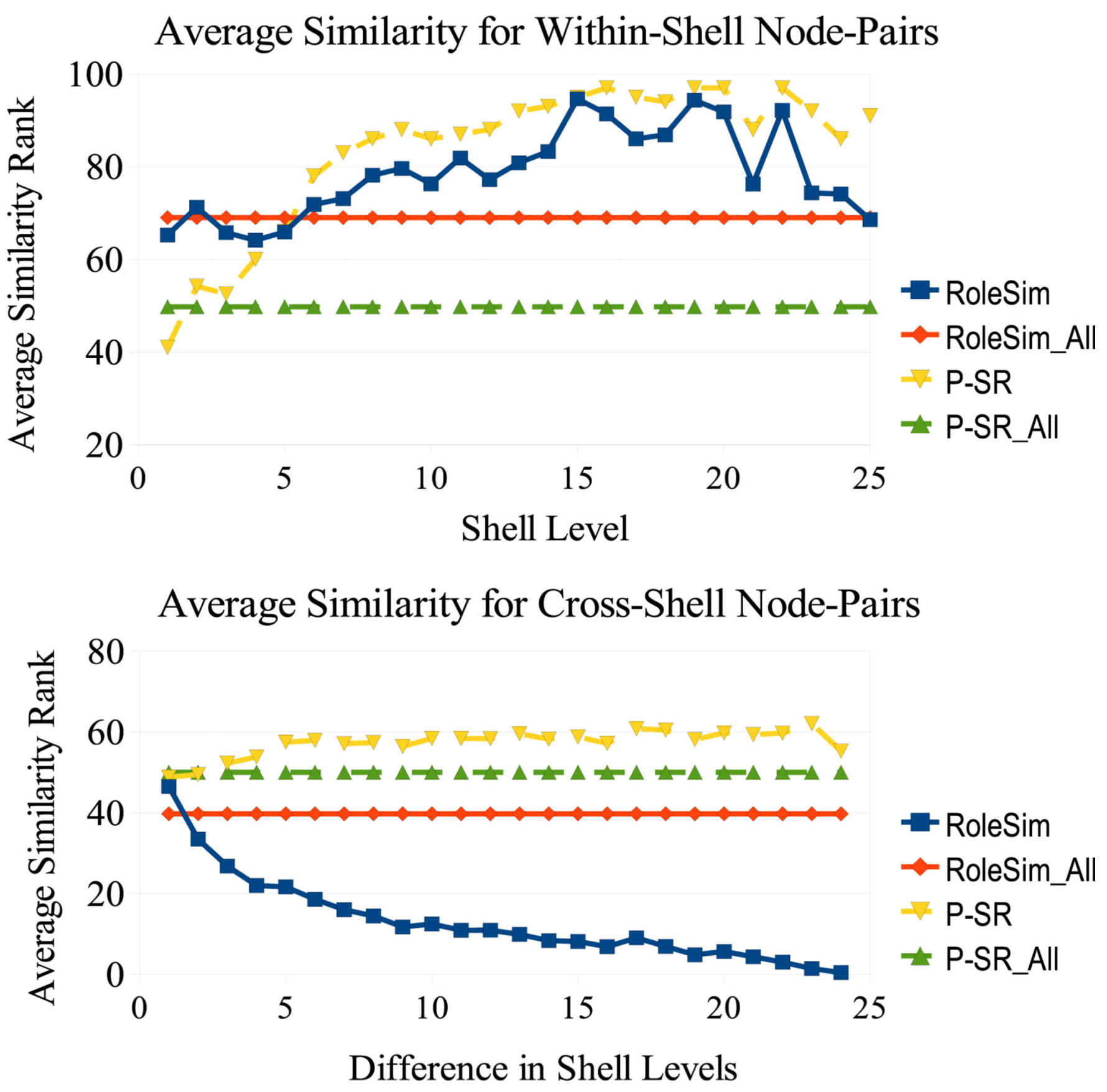

Fig. 8.

Similarity of Authors Grouped By K-Shell 

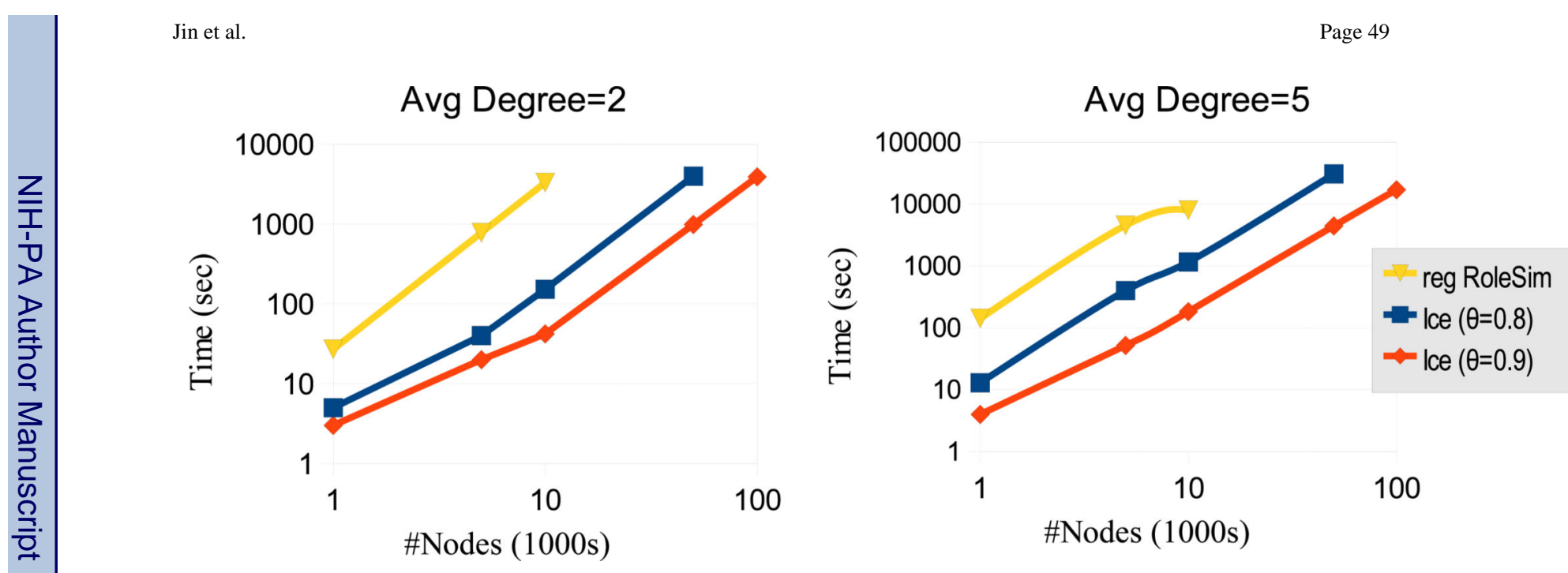

Fig. 9.

Execution time: Standard vs. Iceberg 


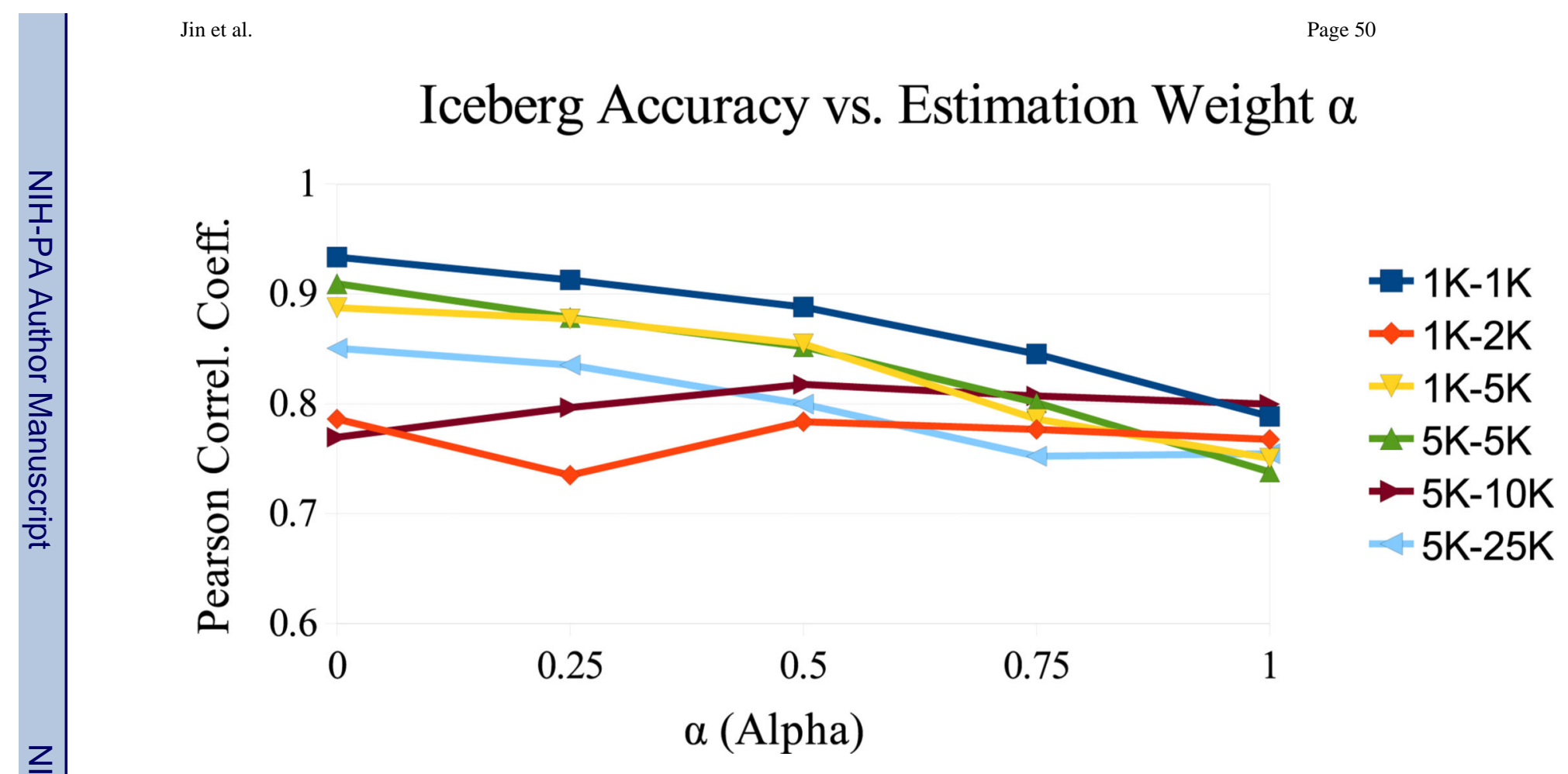

Fig. 10.

Iceberg Accuracy vs. a 


\section{Table I}

Equivalence Classes for Figure 1

\begin{tabular}{|l|l|l|l|}
\hline Equivalence & Neighbor Rule & Non-singleton Classes & Unique Partitioning? \\
\hline Structural & same nodes $(N(u)=N(v))$ & $\{\mathrm{S} 3, \mathrm{~S} 4\},\{\mathrm{J} 3, \mathrm{~J} 4\},\{\mathrm{L} 3, \mathrm{~L} 4, \mathrm{~L} 5\}$ & Yes \\
\hline Automorphic & $\begin{array}{l}\text { For automorphism } \sigma, \forall x \in N(u), \exists y \in \\
N(\mathrm{v}) \text { s.t. } y=\sigma(x)\end{array}$ & $\{\mathrm{S} 1, \mathrm{~J} 1\},\{\mathrm{S} 2, \mathrm{~J} 2\},\{\mathrm{S} 3, \mathrm{~S} 4, \mathrm{~J} 3, \mathrm{~J} 4\},\{\mathrm{L} 3, \mathrm{~L} 4, \mathrm{~L} 5\}$ & Yes \\
\hline Equitable Partition & same number per class & $\{\mathrm{S} 1, \mathrm{~J} 1\},\{\mathrm{S} 2, \mathrm{~J} 2\},\{\mathrm{S} 3, \mathrm{~S} 4, \mathrm{~J} 3, \mathrm{~J} 4\},\{\mathrm{L} 3, \mathrm{~L} 4, \mathrm{~L} 5\}$ & No \\
\hline Regular & same classes & $\{\mathrm{S} 1, \mathrm{~J} 1, \mathrm{~L} 1\},\{\mathrm{S} 2, \mathrm{~J} 2, \mathrm{~L} 2\},\{\mathrm{S} 3, \mathrm{~S} 4, \mathrm{~J} 3, \mathrm{~J} 4, \mathrm{~L} 3, \mathrm{~L} 4, \mathrm{~L} 5\}$ & No \\
\hline
\end{tabular}




\section{Table II}

Structural Similarity Measures

\begin{tabular}{|c|c|}
\hline Measure & Formula \\
\hline bibliographic coupling & $S_{b c}(a, b)=\frac{|O(a) \cap O(b)|}{|O(a) \cup O(b)|}$ \\
\hline co-citation & $S_{c c}(a, b)=\frac{|I(a) \cap I(b)|}{|I(a) \cup I(b)|}$ \\
\hline cosine & $S_{\cos }(a, b)=\frac{A \cdot B}{\|A\|\|B\|}$ \\
\hline Tanimoto & $S_{\text {tani }}(a, b)=\frac{A \cdot B}{\|A\|^{2}+\|B\|^{2}-A \cdot B}$ \\
\hline weighted cosine & $S_{w \cos }(a, b)=\frac{\sum_{t \in T} A(t) B(t) i d f(t)}{\|A\|\|B\|}$ \\
\hline SimRank & $\operatorname{sim}_{s r}(a, b)=\frac{c}{|I(a)| I(b) \mid} \sum_{x \in I(a)} \sum_{y \in I(b)} \operatorname{sim}_{s r}(x, y)$ \\
\hline SimRank++ & $\operatorname{sim}_{s p p}(a, b)=c\left(\sum_{i=1}^{|N(a) \cap N(b)|} \frac{1}{2^{i}}\right) \sum_{x=1}^{N(a)} \sum_{y=1}^{N(b)} w_{a b} w_{b y} \operatorname{sim}_{s p p}(x, y)$ \\
\hline PSimRank & $\operatorname{sim}_{p s}(a, b)=c\left(\frac{|I(a) \cap I(b)|}{|I(a) \cup I(b)|}+\frac{\sum_{\substack{x \in I(a) \backslash I(b), y \in I(b)}} \operatorname{sim}_{p s}(x, y)}{|I(a) \cup I(b)||I(b)|}+\frac{\sum_{\substack{x^{\prime} \in I(b) \backslash I(a), y^{\prime} \in I(a)}} \operatorname{sim}_{p s}\left(x^{\prime}, y^{\prime}\right)}{|I(b) \cup I(a)||I(a)|}\right.$ \\
\hline MatchSim & $\operatorname{sim}_{m s}(a, b)=\frac{\sum_{(x, y) \in m_{a b}^{\star}} \operatorname{sim}_{m s}(x, y)}{\max (|I(a)|,|I(b)|)}$ \\
\hline PageSim & $\operatorname{sim}_{p g 2}(a, b)=\frac{\sum_{i=1}^{n} \min \left(F V_{i}(a), F V_{i}(b)\right)}{\sum_{i=1}^{n} \max \left(F V_{i}(a), F V_{i}(b)\right)}$ \\
\hline VertexSim & $D S_{v} D=\frac{c}{\lambda_{1}} A\left(D S_{v} D\right)+I$ \\
\hline
\end{tabular}


Table III

Properties of Similarity Measures

\begin{tabular}{|l|l|l|l|}
\hline Similarity Measure & $\begin{array}{l}\text { Automorphism } \\
\text { Confirmation }\end{array}$ & Transitivity & Similarity Ordering \\
\hline bibliographic coupling & No & Yes & Yes \\
co-citation & No & Yes & Yes \\
SimRank & No & - & - \\
SimRank++ & No & - & - \\
PSimRank & No & - & - \\
MatchSim & No & Yes & Yes \\
PageSim & Yes & Yes & No \\
VertexSim & No & - & - \\
\hline
\end{tabular}


Table IV

Performance Comparison of Initialization Methods

\begin{tabular}{|c|c|c|c|c|}
\hline \multirow{2}{*}{$\begin{array}{l}\text { Performance Relative to } \\
\text { ALL-1 Initialization }\end{array}$} & \multirow{2}{*}{$\begin{array}{l}\text { Degree-Ratio } \\
\text { Init. }\end{array}$} & \multicolumn{3}{|c|}{ Degree-Binary Init. } \\
\hline & & Min. & Avg. & Max. \\
\hline Difference in percentile rank & none & $0.14 \%$ & $0.38 \%$ & $11.17 \%$ \\
\hline Pearson correlation coefficient & 1 & 0.9994 & 0.9998 & 0.9999 \\
\hline Relative execution time & $\approx 0.9$ & 0.32 & 0.52 & 0.80 \\
\hline Relative \# iterations & 1 fewer & 0.38 & 0.58 & 0.88 \\
\hline
\end{tabular}


Table V

Iceberg Size Relative to RoleSim Matrix

\begin{tabular}{|c|c|c|}
\hline \multirow{2}{*}{$\begin{array}{l}\text { Edge Density } \\
(|E| /|V|)\end{array}$} & \multicolumn{2}{|c|}{ Iceberg Size, as fraction of full matrix } \\
\hline & $\theta=\mathbf{0 . 8}$ & $\theta=\mathbf{0 . 9}$ \\
\hline 1 & $2.77 \%$ & $1.47 \%$ \\
\hline 2 & $2.47 \%$ & $0.63 \%$ \\
\hline 5 & $3.53 \%$ & $0.15 \%$ \\
\hline
\end{tabular}




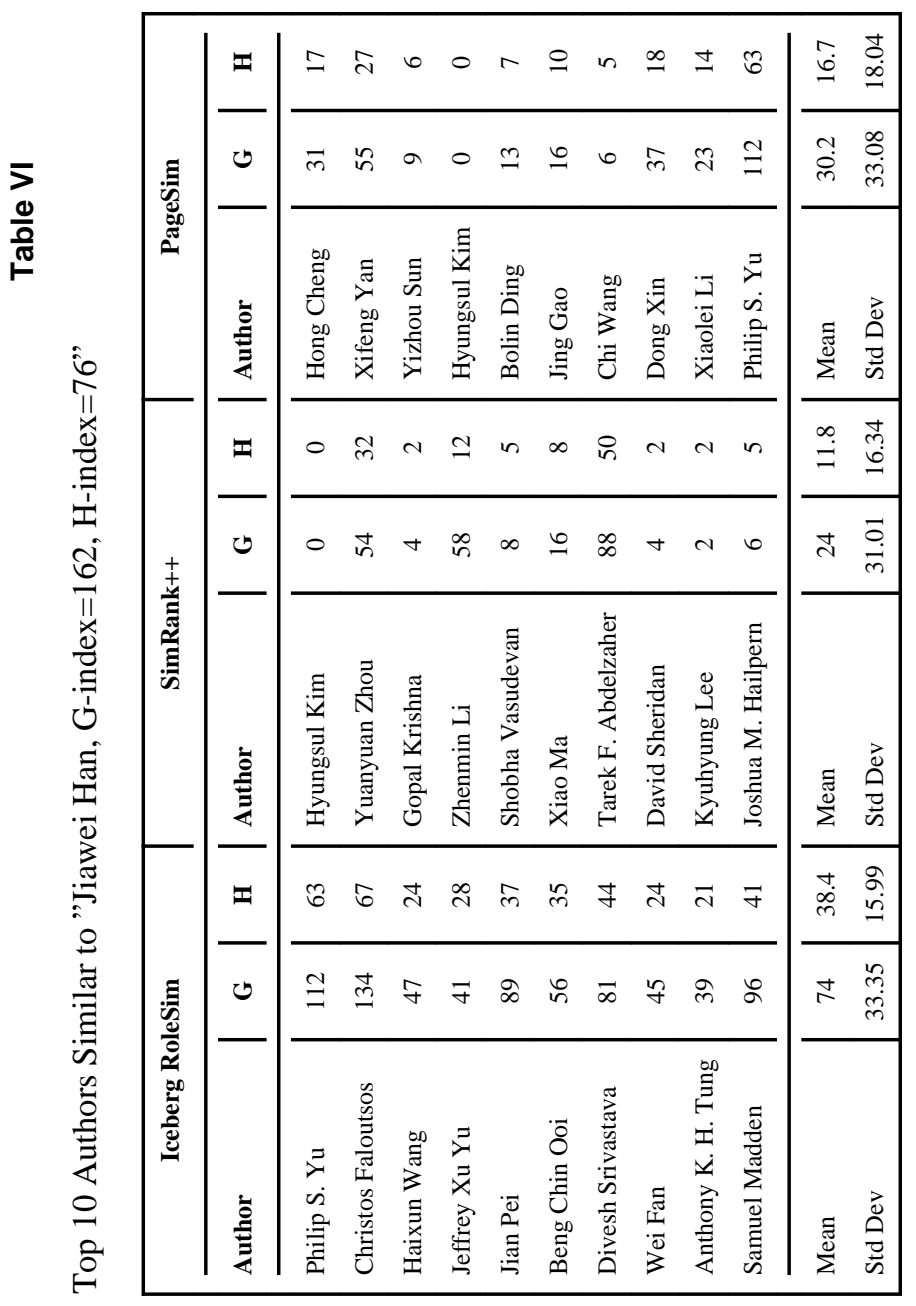




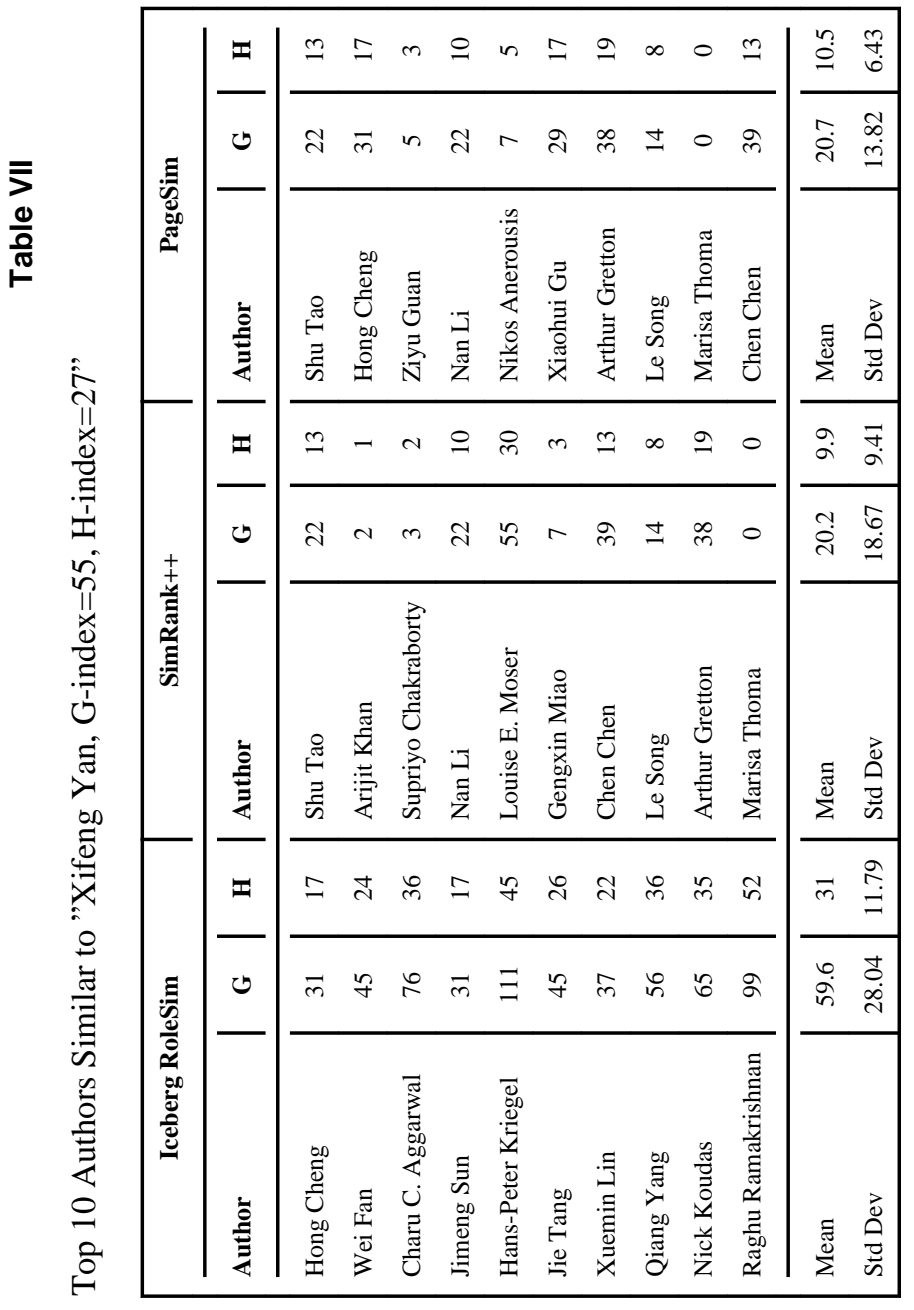




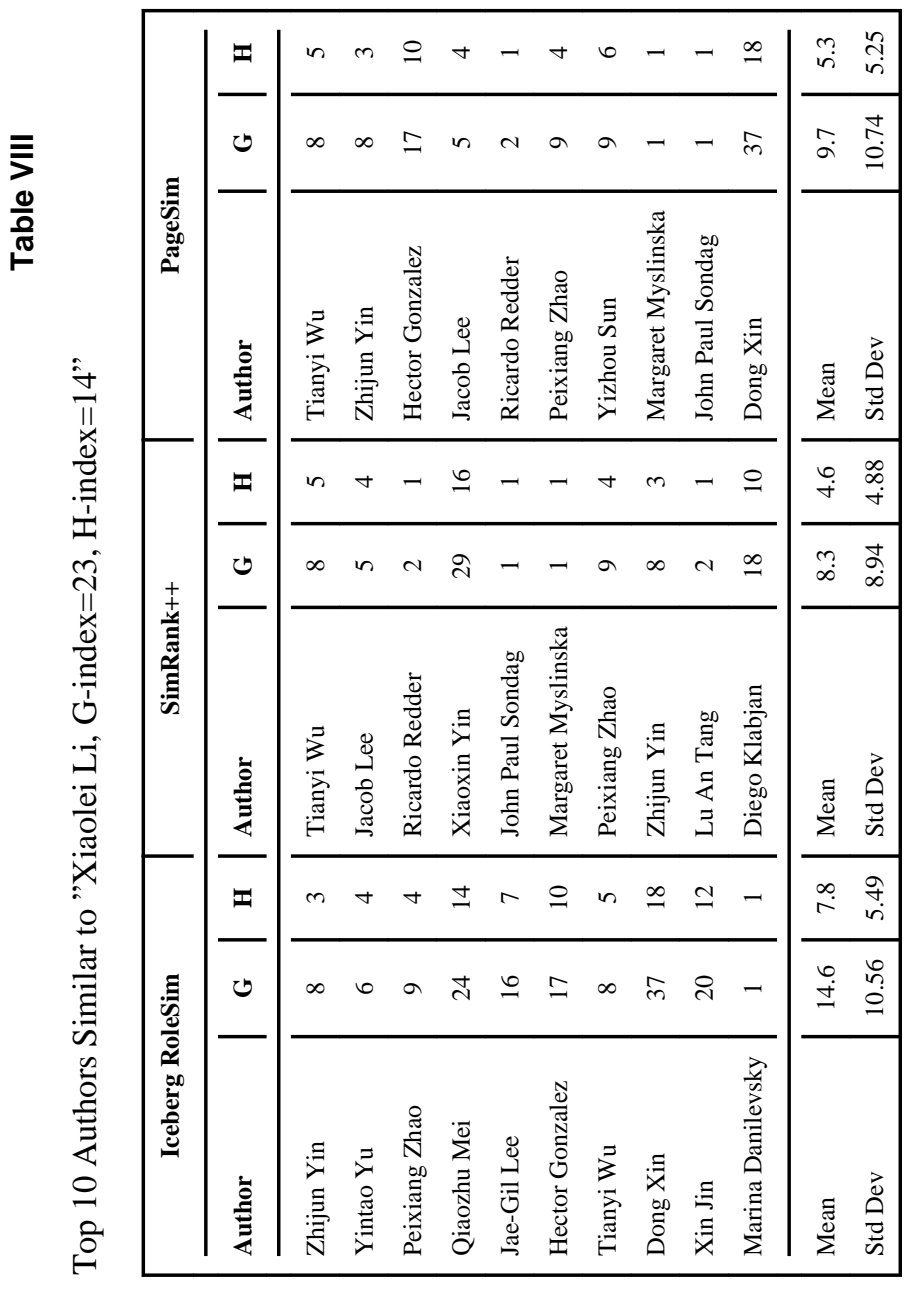

ACM Trans Knowl Discov Data. Author manuscript; available in PMC 2014 November 06. 